\title{
MAGNETIC ASPECTS OF SUPERCONDUCTING PAIRING
}

\author{
M. ACquarone \\ C.N.R., Dipartimento di Fisica dell' Universitá, 43100 Parma, Italy \\ and M. Paiusco \\ I.N.F.M., Dipartimento di Fisica dell' Universitá, 43100 Parma, Italy
}

\begin{abstract}
We discuss some of the recently proposed models dealing with the superconducting state in the presence of strong antiferromagnetic fluctuations, induced by the on-site Coulomb repulsion between electrons (the Hubbard correlation). In the weak coupling limit we treat the influence of correlation effects on the phonon-mediated superconductivity within the BCS-Eliashberg framework. As a non-standard model in this limit we describe next the so-called spin bag model. To discuss strong correlation limit, we describe first the projected Hamiltonian (the $t-J$ Hamiltonian) and the corresponding antiferromagnetic state. The resonating valence bond model is introduced both as a naive version, equivalent to the BCS treatment of the transverse spin fluctuations, as well as a holon-spinon version.
\end{abstract}

PACS numbers: 74.10.-z, 75.10.Lp, 75.50.Ee

\section{The weak correlation limit}

\subsection{Outline of the antiferromagnetic state}

Magnetically ordered states are due to the repulsion between electrons, measured by the on-site electron correlation energy $U$. We shall use the Hubbard IIamiltonian for a single band in the site and reciprocal space representation

$$
\begin{aligned}
& H=\sum_{i j \sigma} t_{i j} a_{i \sigma}^{\dagger} a_{j \sigma}+\frac{U}{2} \sum_{i \sigma} n_{i \sigma} n_{i-\sigma}, \\
& H=\sum_{k, \sigma} \varepsilon_{k} a_{k \sigma}^{\dagger} a_{k \sigma}+\frac{U}{2 N} \sum_{k p q q_{\sigma}} a_{k+p, \sigma}^{\dagger} a_{k, \sigma} a_{q-p,-\sigma}^{\dagger} a_{q,-\sigma} .
\end{aligned}
$$

The local moment $S_{i}$ has components $\left\{S_{i}^{+}, S_{i}^{-}, S_{i}^{z}\right\} \equiv\left\{a_{i \uparrow}^{\dagger} a_{i \downarrow}, a_{i \downarrow}^{\dagger} a_{i \uparrow},\left(n_{i \uparrow}-n_{i \downarrow}\right) / 2\right\}$. In the antiferromagnetic (AF) state, the Fourier transform of $S_{i}^{z}$ i.e. $S_{q}^{z} \equiv(1 / N) \sum_{i} S_{i}^{z} \exp \left(-\mathrm{i} \boldsymbol{q} \cdot \boldsymbol{R}_{\boldsymbol{i}}\right)$ can be written

$$
S_{\boldsymbol{q}}^{z}=\frac{1}{2 N} \sum_{k_{\sigma}} \sigma a_{k_{\sigma}}^{\dagger} a_{k_{+}+q_{\sigma}}
$$


For a Néel-type (up-down) of order, $q=G / 2$ with $G-$ a reciprocal lattice veclor. In the Hartree-Fock approximation the IIubbard Hamiltonian becomes

$$
H=\sum_{k_{\sigma}}\left(\varepsilon_{k}+\frac{1}{2} U n\right) a_{k_{\sigma}}^{\dagger} a_{k_{\sigma}}-U \sum_{k_{\sigma}} \sigma S_{q}^{z}\left(a_{k_{\sigma}}^{\dagger} a_{k+q_{\sigma}}+a_{k_{+}+q_{\sigma}}^{\dagger} a_{k_{\sigma}}\right) .
$$

It can be diagonalized by introducing new operators by means of a Bogolyubov transformation. If $p$ is any $k$ vector such that $|k|<|G / 2|$, by substituting in (1.1.3)

$$
\begin{aligned}
& a_{p_{\sigma}}^{\dagger} \equiv c_{1 p_{\sigma}}^{\dagger} \cos \theta_{p_{\sigma}}-c_{2 p_{\sigma}}^{\dagger} \sin \theta_{p_{\sigma}}, \\
& a_{p_{+} q_{\sigma}}^{\dagger} \equiv c_{1 p_{\sigma}}^{\dagger} \sin \theta_{p_{\sigma}}-c_{2 p_{\sigma}}^{\dagger} \cos \theta_{p_{\sigma}},
\end{aligned}
$$

we obtain the AF Hamiltonian in diagonal form

$$
H^{\mathrm{AF}}=\sum_{p_{\sigma}}\left(E_{1} p c_{1}^{\dagger} p_{\sigma} c_{1} p_{\sigma}+E_{2 p} c_{2}^{\dagger} p_{\sigma} c_{2} p_{\sigma}\right)
$$

provided $\tan \left(2 \theta_{p_{\sigma}}\right)=\sigma U S_{q}^{z} /\left(\varepsilon_{q}-\varepsilon_{p+q}\right)$. The $A F$ quasi-particle encrgics for materials exhibiting "perfect nesting" of the band structure, i.e. $\varepsilon_{p}=-\varepsilon_{p+G / 2}$ (also called the folding condilion) are

$$
E_{1,2}=\frac{U n}{2} \pm \sqrt{\varepsilon_{p}^{2}+\left(U S_{q}^{z}\right)^{2}} .
$$

Two AF subbands result, separated by a gap $\Delta=2 U S_{q}^{z}$. At the gap edges, the AF density of states (DOS) has spikes diverging to infinity. The AF gap for a spin density wave vector $q=G / 2$ opens in the middle of the paramagnetic (PM) band irrespective of $n$. The value of $S_{q}^{z}$ (hence of the gap $\Delta \equiv 2 U S_{q}^{z}$ ) can be evaluated explicitly for any band occupancy $n$ in the case of a PM square DOS $\rho_{\sigma}=1 / W$, yielding

$$
S_{\boldsymbol{q}}^{z}=\frac{1}{U \operatorname{Sh}(W / U)} \sqrt{\varepsilon_{\mathbf{L}}^{2}+\varepsilon_{\mathrm{F}}^{2}(n)-2\left|\varepsilon_{\mathrm{L}} \varepsilon_{\mathrm{F}}(n)\right| \operatorname{Ch}(W / U)},
$$

while for the square bidimensional lattice one has the implicit equation

$$
S_{\boldsymbol{q}}^{z}=\frac{2 W / U}{\sqrt{1+\left[\varepsilon_{\mathrm{F}}(n) / U S_{q}^{z}\right]^{2}}} \exp (-\pi \sqrt{W / 2 U}) .
$$

In the relation above, $\varepsilon_{\mathrm{L}}$ and $\varepsilon_{\mathrm{F}}(n)$ are respectively the lower band edge and the Fermi level in the PM state. In both cases $S_{\boldsymbol{q}}^{z}$ decreases quickly with $W / U$ and $1-n$. By comparing the above equations, one sees that the sensitivity of $S_{\boldsymbol{q}}^{z}$ to variations of $W / U$ and the filling $n$ is smaller for the square lattice DOS, characterized by the van Hove singularity.

\subsection{Phononic superconductivily in a weakly correlated AF background}

This topic would require a rather detailed analysis which we cannot do here. For a very detailed discussion, see [1]. We shall instead briefly point out the main problems in the framework of the standard models of phonon-assisted superconductivity (SC).

According to the BCS theory [2] the expression of $T_{\mathrm{c}}$ assuming a model DOS appropriate to free electrons in three dimensions is

$$
k_{\mathrm{B}} T_{\mathrm{c}}=\Delta_{\mathrm{D}} / 3.53=1.33 \hbar \omega_{\mathrm{D}} \mathrm{e}^{-1 / \rho^{N}\left(E_{\mathrm{F}}\right) V},
$$


where $\Delta_{\mathrm{D}}$ is the SC gap in the density of states, $\omega_{\mathrm{D}}$ is the Debye frequency, $\rho^{N}\left(E_{\mathrm{F}}\right) \equiv \rho_{\mathrm{F}}^{N}$ is the DOS at the Fermi level in the state existing at $T \rightarrow T_{\mathrm{c}}^{\dagger}$ (normal state). $V$ is the matrix element of the electron-phonon coupling responsible for the pairing of the electrons, which BCS assumed to be isotropic on the Fermi surface for convenience. In general, $V \equiv V_{k, q}$ i.e. it depends on the wave vectors of the electron $(k)$ and of the phonon $(q)$ involved. In that case, one must distinguish between two gaps. One type of gap $\Delta_{k}$ separates states with the same $k$ in the upper $(+)$ and lower $(-)$ SC subbands

$$
\Delta_{k} \equiv E_{k}^{+}-E_{k}^{-} \text {. }
$$

The other type of gap $\Delta_{\mathrm{D}}$ is the gap in the resulting SC density of states

$$
\Delta_{\mathrm{D}} \equiv \operatorname{Min}\left\{E_{k}^{+}\right\}-\operatorname{Max}\left\{E_{k}^{-}\right\} \text {. }
$$

This distinction is of great importance when one treats the effect of impurities [2]. If not stated explicitly otherwise, we shall consider only the isotropic case (s-wave coupling), where $\Delta_{k}$ is actually $k$-independent and the two gaps coincide $\Delta_{k}=\Delta_{\mathrm{D}}$.

The BCS theory neglects the effect of correlation, and therefore it is not a good starting point to deal with magnetic effects on the SC state, because, as we have seen, magnetism is due to appreciable on-site repulsion effects. If the SC state evolves from a PM state, correlation has two effects. The first one is to reduce the strength of the electron-phonon coupling in a way approximately proportional to the reduction in the cohesive energy [3]. The second effect affects the efficiency of a given electron-phonon interaction in coupling the electrons. This effect is described by the Eliashberg theory [4]. Therefore, one cannot write down a simple expression for $T_{\mathrm{c}}$, but one can find an approximate solution for the DOS gap $[5,6]$ :

$$
\begin{aligned}
& 1=\frac{\rho^{N}(0)}{2}\left[\lambda-\frac{\lambda \mu^{*} \log \left(\varepsilon_{\mathrm{F}} / \omega_{\mathrm{D}}\right)}{2}-\frac{\mu^{*}}{\rho^{N}(0)}\right] \log \left(\frac{2 \omega_{\mathrm{D}}}{\Delta_{\mathrm{D}}}\right), \\
& \mu^{*} \equiv \frac{U \rho^{N}(0)}{1+\left[\rho^{N}(0) U / 2\right] \log \left(\varepsilon_{\mathrm{F}} / \omega_{\mathrm{D}}\right)} .
\end{aligned}
$$

The quantity $\mu^{*} / \rho^{N}(0)$ just defined is the effective Coulomb correlation, which is strongly reduced with respect to the bare $U$ whenever $\varepsilon_{\mathrm{F}} \gg \omega_{\mathrm{D}}$. The physical process (usually termed "retardation effect") is the following. In metals where $U \ll W$, one usually has $\hbar \omega_{\mathrm{D}} \ll E_{\mathrm{F}}$, implying that the transit time of the electrons in any given lattice cell $\left(\tau_{\mathrm{el}} \approx h / E_{\mathrm{F}}\right)$ is much shorter than the ion vibration period $\left(\tau_{\text {Ion }} \approx 2 \pi / \omega_{\mathrm{D}}\right)$. Then the electron which triggers the vibration of a given ion is already very far away when a second electron, passing close to the same ion, feels the vibration and gets coupled to the first electron. In this way, the effective repulsion between the coupled electrons is much reduced with respect to $U$, which is defined as the instantaneous (unretarded) repulsion occurring when both electrons are around the same ion. In the present case, usually their relative distance at the moment they get coupled, being $\approx v_{\mathrm{F}} \tau_{\text {ion }}$, is much larger than a lattice distance $a \approx v_{\mathrm{F}} \tau_{\mathrm{el}}$. Of course, if the Fermi energy is strongly reduced, or the phonon vibration frequency strongly enhanced, this argument breaks down.

However, it turns out that even in that case, which invalidates the adiabatic approximation and the Migdal theorem [4], the effective correlation is well screened [7]. 
When the correlation is strong enough that the normal state is not PM but AF (or, at least, a PM state with long lived magnetic fluctuations of appreciable amplitude which create islands of AF order extending over distances large in comparison to $k_{\mathrm{F}}^{-1}$ ) one might argue that such a state has some features, connected to the renormalized density of states, which might be favourable to singlet SC. Indeed, we have seen that the AF DOS develops peaks close to the AF gap. By consequence, when the filling is close to, but less than one, so that $E_{\mathrm{F}}$ falls close to the gap, $\rho^{\mathrm{AF}}\left(E_{\mathrm{F}}\right) \gg \rho^{\mathrm{PM}}\left(E_{\mathrm{F}}\right)$ and an enhancement of $T_{\mathrm{c}}$ might be expected $[8,9]$. On the other side, the Coulomb interaction itself should be more efficiently screened in the AF than in the PM state. Indeed, the Thomas-Fermi screening length in the low- $U$ limit goes as the inverse of the density of states at the Fermi energy, being therefore larger in the PM than in the AF phases for $n \approx 1$. This reasoning, however, neglects the generally greater stability of the AF state with respect to the singlet $\mathrm{SC}$ one because the $\mathrm{AF}$ gap goes as $2 U S_{q}^{z} \approx U$, while the phonon-assisted SC gap is smaller than $\hbar \omega_{\mathrm{D}} \ll U$. Of course, this argument depends on the actual $U / W$ and $U / \hbar \omega_{\mathrm{D}}$ values, so that it has to be checked for a given material.

A more relevant feature of the problem is that the interactions (e.g. on-site correlation, electron-phonon etc.) present in the PM Hamiltonian have to be re-expressed in terms of the quasi-particle operators appropriate to the normal non-PM state (the spin density wave (SDW) state in this case) out of which the SC state is assumed to develop. For instance, the electron-phonon interaction term has in the PM state the expression [6]:

$$
H_{\mathrm{ep}}=\sum_{\sigma} \sum_{k q_{\nu}} g_{k q^{\prime}}^{\nu}\left(b_{q_{\nu}}+b_{-q_{\nu}}^{\dagger}\right) a_{k_{+q, s}}^{\dagger} a_{k_{\nu}},
$$

where $\nu$ is the polarization index and the remaining notation is standard. The form appropriate to study the possibility of the phonon-mediated SC state in a background with AF fluctuations is obtained from Eq. (1.2.6) by substituting to each fermion operator $a_{k_{\sigma}}$ either one of the AF operators $c_{p \sigma}$ or $c_{p+G / 2, \sigma}$ according to Eq. (1.1.4). The results $[1,10,11]$ obtained by neglecting the dependence of the coupling on the phonon wave vector $q$, indicate a decrease in the coupling for singlet states due to the SDW background. However, a different conclusion (i.e. that correlation enhances the electron-phonon interaction) is reached in [12] where the $q$ dependence was explicitly considered. The same conclusion was reached in the strongly correlated case in [13]. A fully convincing treatment is still lacking, but any conclusion about appreciably correlated systems reached by total neglect of such effects on the interactions [14-16] are likely to be unreliable.

\subsection{Non-phononic superconductivily in a weakly correlated AF background}

Besides the electron-phonon interaction, also the correlation term itself has to be expressed in terms of the AF quasi-particle operators. The consequences are the core of the spin bag mechanism of SC proposed in the context of the high- $T_{c}$ superconductors by Schrieffer and co-workers [17].

Referring to the quoted papers for discussion of the experimental evidence underlying the model, we shall recall its basic ideas only. The starting point is 
the observation that the SDW remains commensurate as holes are doped into the material, implying that the AF subband structure is essentially rigid, so that when $n<1$ one has a metallic AF. Indeed, if the SDW pitch varied with $n$, as soon as $n<1$ it would become incommensurate with the lattice, and the Fermi energy, for any $n$, would be pinned at the gap edge, keeping the material in the insulating AF state. The holes are considered to be represented by wave packets of size $l \ll \xi_{\text {SDW }}$, where $\xi_{\text {SDW }}$ is the coherence length of the SDW, i.e. the distance over which two spins keep an appreciable AF correlation. For a long-range ordered AF state, $\xi_{\text {sDW }} \rightarrow \infty$, while if the macroscopic magnetic state is PM, then $\xi_{\text {SDW }}$ can be $\approx 10-10^{2}$ lattice spacings (e.g. in $\mathrm{La}_{2-x} \mathrm{Sr}_{x} \mathrm{CuO}_{4}$, the precise value depending on $x$ ). This introduces a self-consistency requirement into the theory for the superconducting coupling, because the calculated hole pairing potential $V_{\boldsymbol{p}}$ must turn out to be large for a range $\delta p$ of vectors of the reduced Brillouin zone (RBZ) such that $\delta p \approx l^{-1}$. The condition over $l$ implies that the $A F$ band structure around the position of the hole is well defined even in the presence of the holes. Actually, Schrieffer and co-workers use the term $S D W$ gap, stressing the short-range order, and fluctuating, nature of the state they consider, instead of $A F$ gap, which refers to long-range and static order. We shall use the two names interchangeably.

An intuitive picture of the pairing is the following: a hole depresses locally the AF order in two ways. First, via exchange interaction, its spin pushes all the surrounding $\mathrm{Cu}^{2+}$ spins to assume the same spatial direction, producing a ferromagnetic region. Notice that the fact that a moving hole carries a spin is a distinctive feature of the low correlation limit. Indeed, in the opposite limit $U>W$, as we shall see later on, the itineracy of the charge (hopping of a hole between two neighbouring sites) has an energy cost of the order $U / W$, i.e. larger than the itineracy of the spin (analogous, but not equal, to the creation of a spin wave) whose cost is of the order $J=W^{2} / U \ll U / W$. In the large correlation limit one has to distinguish between charge and spin motion, as we shall see. This first effect is at the centre of the spin-polaron mechanism for non-degenerate bands discussed in [18] which we cannot comment here. Notice that, under the same name of spin polaron, a quite different mechanism, possible only if the many-band nature of the electronic structure in high- $T_{\mathrm{c}}$ superconductors (IITCSC) materials is taken into account, has been proposed [19]. The second effect depressing the AF order is that the hole moves the Fermi level farther from the band edge, reducing the local $S_{q}$, and, by consequence, the AF gap (see Eqs. (1.1.7) or (1.1.8)). At the same time, the energy gain due to itineracy of the AF particle $\left(\approx E_{\mathrm{F}}(n)-E_{\mathrm{L}}\right)$ is reduced, so that the hole tends to be trapped in the perturbed region: a spin bag has been formed. It is now rather obvious that two such bags minimize their overall energy by sticking together.

Notice that the effective potential keeping the holes inside the bags will now have a range of the order $2 l$ (because each hole is trapped everywhere inside the two-bag region) which reduces the effective Coulomb repulsion between the holes. The quantitative details of the model require rather lengthy calculations, which are exposed quite clearly in the quoted literature.

We shall simply summarize the main steps. Looking back to Eq. (1.1.3) we 
see that the correlation term describes the coupling of PM electron states $k$ and $k+q$ by $U S_{q}$. This interaction is written as appropriate to the ground state, and therefore $q=G / 2$. When excitations (i.e. spin waves) are present, the AF moment will acquire small, fluctuating components of all wave vectors belonging to the RBZ. The fluctuations affect both the amplitude and the orientation of the local moment: the first ones (longiludinal fluctuations) change $\left\langle S_{i}^{z}\right\rangle$, the other ones (Iransverse fluctuations) develop non-vanishing $\left\langle S_{i}^{+}\right\rangle$or $\left\langle S_{i}^{-}\right\rangle$components. There will be also charge excitations, connected with electron hopping.

Schrieffer and co-workers [17] evaluate the different terms of the resulting two-particle interaction in RPA approximation by using the AF operators to describe the fermions involved. They find that, if the holes occupy small pockets near the top of the lower AF band (as it should be the case in the high- $T_{\mathrm{c}}$ superconductors), then the only excitations effective in the coupling are the amplitude fluctuations. They can now couple electrons of opposite spins (because they carry one quantum of spin deviation) and of opposite moments (because they can carry a wave vector spanning the whole RBZ), as required to form a Cooper pair of singlet type. The coefficient of such terms, which is the hole pairing potential, turns out to be negative. Therefore, the model yields a net attraction between the interacting fermions, provided the hole doping is low. The pairing potential $V_{p}(\omega)$ depends on energy (and wave vectors) and has two cut-off frequencies. One is the maximum energy of the amplitude fluctuation itself, which turns out to be approximately equal to the AF gap. The second cut-off is at $\omega=-\Delta / 2-E_{\mathrm{F}}$ in our notation, i.e. it corresponds to the Fermi energy for the holes, evaluated downward from the top of the lower AF subband (i.e. from the gap edge). This follows from the impossibility of exciting electrons above the AF gap edge. The physically relevant cut-off is the lower between the two. A third cut-off refers to the moments, and it follows from the above-mentioned self-consistency requirement, i.e. that the coupled-hole wave packet in the real space has to be contained inside a distance not larger than $\xi_{\text {sDw. }}$. This is guaranteed provided the moment exchanged through the fluctuation $q \geq q_{\min }=0.5 / \xi_{\mathrm{SDW}}$, which, in a free-electron-like formula, corresponds to a minimum energy $\omega_{\min }=\hbar^{2} / 2 m \xi_{\mathrm{SDW}}^{2}$. If we have very few holes, their Fermi energy $\varepsilon_{\mathrm{F}} \equiv-\left(E_{U}-E_{\mathrm{F}}\right)$ can be too small to satisfy this requirement.

To solve the difficulty one can heuristically associate to the holes an effective mass $m^{*}>m$ so that $\omega_{\min }=\hbar^{2} / 2 m^{*} \xi_{\mathrm{SDW}}^{2} \leq \varepsilon_{\mathrm{F}}$. This is a lower bound on the coupling energy, which is expected to be greater than the Debye energy $h \omega_{\mathrm{D}} \approx c q_{\mathrm{D}}$ ( $c$ is the velocity of sound and $q_{\mathrm{D}}$ - the Debye wave vector), because the phonon wavelengths $\lambda_{\mathrm{ph}}>q_{\mathrm{D}}^{-1}$ are generally larger than $\xi_{\mathrm{SDW}}$. By consequence, the spin bag model satisfies the general condition established in [20] for consistency of any non-phononic coupling, which states that the relevant energy scale must be larger than $\hbar \omega_{\mathrm{D}}$. It is important to notice that the on-site Coulomb repulsion is not expected to have a large effect, due to the fact that the opposite spin holes tend to live on different sublattices. This is a real space picture of the mentioned effective screening of the $U$ term in the Thomas-Fermi approximation. Indeed, having $n \approx 1$ puts the AF Fermi level close to the gap, i.e. where the DOS is large and the screening length is small. By consequence (see Eq. (1.1.8)), if $U / W$ is not too small, one has fully developed staggered moment $S_{q} \approx n / 2$ on the two 
sublattices, i.e. a situation close to the Néel order of localized moments. This is tantamount to say that the "minority" carriers (i.e. those with the wrong spin with respect to the sublattice on which they are) are very lew. As the Coulomb repulsion is felt only when opposite spin carriers come onto the same site, if such events are very rare, the overall repulsion effect is small.

The SC gap turns out to be anisotropic in $k$ space, and it has lines, along which it vanishes. However, this has no effects on the experimental quantities which behave differently according to the existence, or absence, of a gap in the DOS (a typical example being the specific heat below $T_{c}$ ), because even if the SC gap vanishes for some $k$ values, the AF gap does not. The basic difference between the two gaps is that the one due to SC is tied to the Fermi surface, so that it may depend on the filling, while the AF gap, being dictated by the symmetry of the lattice and the pitch of the SDW, is independent of the filling, but for the case where a variation of the latter makes it energetically favourable to change also the former. The present model assumes that the SDW wavelength does not change with filling, so that the AF gap is never vanishing and therefore the SC DOS will always show a gap.

The order parameter $\left\langle c_{i \uparrow} c_{j \downarrow}\right\rangle$ can be evaluated explicitly, and one finds that it changes sign for a rotation of $\pi / 2$, similar to a $d$-wave pairing. Therefore, diamagnetic impurities can have a large negative effect, as in $d$-wave SC. The SC gap at zero temperature $\Delta_{\text {SC }}$ turns out to depend on the SDW gap $\Delta_{\text {SDW }}$ according to [17]:

$$
\Delta_{\mathrm{SC}}(0)=\Delta_{\mathrm{SDW}} \exp \left(-1 / \rho\left(E_{\mathrm{F}}\right) V_{k_{\mathrm{F}}}^{z} m_{k_{\mathrm{F}}}^{2}\right),
$$

where $\rho\left(E_{\mathrm{F}}\right)$ is the AF DOS at the AF Fermi level, and $V_{\boldsymbol{k}_{\mathrm{F}}}^{z} m_{\boldsymbol{k}_{\mathrm{F}}}^{2} \equiv U^{*}$ is the effective pairing potential at the Fermi surface, which can be evaluated through

$$
\begin{aligned}
& V_{k_{\mathrm{F}}}^{z} \equiv \frac{U}{1-U \chi_{\Delta_{\mathrm{SDW}}}^{z z}\left(k_{\mathrm{F}}+q\right)}, \\
& \chi_{\Delta_{\mathrm{SDW}}}^{z z}(q)=\frac{1}{N} \sum_{k} \frac{E_{k} E_{k+q}-\varepsilon_{k} \varepsilon_{k+q}-\Delta_{\mathrm{SDW}}^{2} / 4}{E_{k} E_{k+q}\left(E_{k}+E_{k+q}\right)}, \\
& m_{k, p}^{2} \equiv-\left(\sin \theta_{k} \cos \theta_{p}+\sin \theta_{p} \cos \theta_{k}\right) .
\end{aligned}
$$

On the Fermi surface the last expression rcads

$$
m_{k_{\mathrm{F}}}^{2}=-2 \sin \theta_{k_{\mathrm{F}}} \cos \theta_{k_{\mathrm{F}}}=-\sqrt{\frac{1}{4}-\frac{1}{4}\left(\frac{\left|\varepsilon_{p}-\varepsilon_{p+q}\right|}{\sqrt{\left|\varepsilon_{p}-\varepsilon_{p+q}\right|^{2}+\Delta_{\mathrm{SDW}}^{2} / 4}}\right)^{2}} .
$$

The equations above show that the effective electron-electron interaction $U^{*} \approx-U$. The relation $2 \Delta_{\mathrm{SC}}=3.52 k_{\mathrm{B}} T_{\mathrm{c}}$ holds. The critical temperatures predicted by the spin bag model according to Eq. (1.3.1) are evidently larger than those typical of a classical phononic mechanism, as the relevant energy scale is $\Delta_{\text {SDW }} \approx U \gg \hbar \omega_{\mathbf{D}}$, as noted above. However, Eq. (1.3.1) is difficult to reconcile with the pressure dependence of $T_{\mathrm{c}}$ in the high- $T_{\mathrm{c}}$ superconductors. Indeed, a large body of data [21] points to a generally positive effect of pressure on $T_{c}$, which is difficult to understand on the basis of Eq. (1.3.1). The applied pressure is expected 
to decrease $U / W$, and by consequence also the DOS value and $\Delta_{\mathrm{SDW}}=2 U S_{\boldsymbol{q}}^{z}$, with the overall effect to depress $T_{c}$ as given by Eq. (1.3.1). However, the increase in $\varepsilon_{\mathrm{F}}$ due to the pressure has also a favourable effect as it reduces $\mu^{*}$ according to (1.2.4), possibly overcompensating the reduction of $U / W$ and finally resulting in an enhancement of $T_{\mathrm{c}}$. On the other side, already the starting assumption of the spin bag model, i.e. that the regime $U / W \leq 1$ applies to such materials, is questionable on the same experimental ground, as the Neel temperature $T_{\mathrm{N}}$ in the stoichiometric compounds increases with pressure [17], a fact which suggests that the opposite limit $U / W \gg 1$ is realized at least for $n=1$. One should however mention that recent results on many-band IIamiltonian suggest the possibility of accounting for the pressure data even in the moderately correlated limit [22].

\section{The high-correlation limit}

\subsection{The projected Hamillonian and the $t-J$ Hamillonian}

There has been an enormous amount of interest in the properties of highly correlated systems in connection both with heavy fermion and IITCSC materials. The topics we shall discuss are a tiny sample of the wealth of models and techniques which have been proposed in recent years. The physical problem is that of finding a convenient way of describing the motion of the fermions (when the number of electrons in the system is such that there is on the average no more than one electron per lattice site) under the constraint that the same site cannot be shared by two particles (obviously with opposite spins). A double occupancy would indeed cost an energy $U$ which, in this case, is by assumption larger than $W$, i.e. the energy gained in allowing the particles to freely visit all the sites, only subjected to the Pauli exclusion principle as fully delocalized fermions would do. In the large correlation limit, then, the electrons will tend to avoid each other: either because of the Pauli principle, if they have the same spin, or because of correlation, if the spins are opposite. In the Hilbert space of the Hubbard Hamiltonian in the site representation, where a state is identified by the occupation number of each lattice site, the states with doubly occupied sites will be empty. In that limit if there is exactly one electron per site (half-filled band case), no particle will be able to move, and the electrical resistivity will be that of an insulator with a gap between occupied and empty states of the order of $U$.

The theoretical problem is therefore to find a representation of the Hubbard Hamiltonian in the subspace of states corresponding to configurations with only empty, or singly occupied sites. We shall mainly deal with what is by now the most popular Hamiltonian in this context, the so-called $t-J$ model. It was first introduced by Chao, Spalek, and Oleś [23] under the name of kinetic exchange Hamillonian and then rederived independently by IIirsch [24]. We can recall its derivation from the Hubbard IIamiltonian only in a very sketchy way for lack of space, so we refer the reader to the literature [23-25] for details.

For the physical reasons mentioned above, we shall divide the Hilbert space of the Hubbard Hamiltonian into one subspace containing states with no doubly occupied sites, and another one containing all the remaining states, respectively 
corresponding to the electron levels in the lower and upper Mott-IIubbard subbands. For large $U / W$, a wide gap separates the subbands, and we expect that the matrix elements of the Ilubbard Ilamiltonian connecting the subspaces will be almost negligible. Then it should be possible to substitute to the original Hamiltonian another one resulting of two parts $P_{0} \mathcal{H} P_{0}$ and $P_{\eta} \mathcal{H} P_{\eta}$, each one acting separately on one subspace $(\eta=0$ and $\eta \neq 0)$ and hopefully simpler to handle. We cannot describe the procedure in detail for lack of space, and we refer the reader to the original paper [23]. The final result is

$$
\begin{aligned}
& P_{0} \mathcal{H} P_{0}=\sum_{(i j), \sigma} t_{i j} a_{i \sigma}^{+}\left(1-n_{i-\sigma}\right) a_{j \sigma}\left(1-n_{j-\sigma}\right) \\
& +\sum_{\langle i j\rangle} \frac{2 t_{i j}^{2}}{U}\left[S_{i} \cdot S_{j}-\frac{1}{4} \sum_{\sigma, \tau} n_{i \sigma}\left(1-n_{i-\sigma}\right) n_{j \tau}\left(1-n_{j-\tau}\right)\right] \\
& +\sum_{\langle i j k\rangle} \sum_{\sigma \tau} \frac{t_{i j} t_{j k}}{U} a_{j}^{+} \tau\left(1-n_{j-\tau}\right) a_{k \tau} n_{k-\tau} a_{k \sigma}^{+} n_{k-\sigma} a_{i \sigma}\left(1-n_{i-\sigma}\right), \\
& P_{\eta} \mathcal{H} P_{\eta}=\sum_{\langle i j\rangle, \sigma} t_{i j} a_{i \sigma}^{+} n_{i-\sigma} a_{j \sigma} n_{j-\sigma}+U \sum_{i} n_{i \uparrow} n_{i \downarrow} \\
& +\sum_{\langle i j\rangle} \frac{2 t_{i j}^{2}}{U}\left[n_{i \uparrow} n_{i \downarrow}\left(1-n_{j \downarrow}\right)\left(1-n_{j \uparrow}\right)-a_{i \uparrow}^{+} a_{i \downarrow}^{+} a_{j \downarrow} a_{j \uparrow}\right] \\
& +\sum_{\langle i j k\rangle} \sum_{\sigma \tau} \frac{t_{i j} t_{j k}}{U} a_{j}^{+} \sigma n_{j-\sigma} a_{k \sigma}\left(1-n_{k-\sigma}\right) a_{k \sigma}^{+} a_{i \sigma} n_{i-\sigma},
\end{aligned}
$$

where $\langle i j\rangle$ and $\langle i j k\rangle$ mean that the sites $i, j$ and $k \neq i$ are nearest neighbours. The three-sites processes give a contribution to the real hopping $\approx 1-n$ for $1-n \ll 1$, therefore, for the moment, we shall neglect them. We shall see in the last section that they are connected with the resonating valence bond (RVB) model of high- $T_{\mathrm{c}}$ materials.

We shall consider here only the case $n \leq 1$, because the case $n>1$ can be recovered by an electron $\rightarrow$ hole transformation. Then we concentrate on Eq. (2.1.1), referring to the subspace without double occupancies. We can check immediately that, if $n_{i \sigma}+n_{i}-\sigma=1$, i.e. for the half-filled band case, $P_{0} \mathcal{H} P_{0}$ yields the Heisenberg Hamiltonian. Equation (2.1.1) can be written more compactly by introducing new projected operators $b_{i \sigma}^{+} \equiv a_{i \sigma}^{+}\left(1-n_{i-\sigma}\right)$ and $\nu_{i \sigma} \equiv b_{i \sigma}^{+} b_{i \sigma}=n_{i \sigma}\left(1-n_{i}-\sigma\right)$. The factor $\left(1-n_{i-\sigma}\right)$ forces $b_{i \sigma}^{+}$and $\nu_{i \sigma}$ to be non-vanishing only within the subspace of states with no double occupancy, because if on the site $i$ there is a $-\sigma$ electron, then $1-n_{i-\sigma}=0$, and operating with them yields zero. Then the first two lines of Eq. (2.1.1) can be written as

$$
P_{0} \mathcal{H} P_{0}=\sum_{\langle i j\rangle, \sigma} t_{i j} b_{i \sigma}^{+} b_{j} \sigma+\sum_{\langle i j\rangle} \frac{2 t_{i j}^{2}}{U}\left(S_{i} \cdot S_{j}-\frac{1}{4} \sum_{\sigma} \nu_{i \sigma} \nu_{j-\sigma}\right) .
$$

The kinetic exchange Hamiltonian [23] of Eq. (2.1.3) has the appearance of the IIamiltonian for an uncorrelated single band, where the correlation is hidden into the use of the projected operators $b_{i \sigma}^{+}$and $\nu_{i \sigma}$ instead of the bare operators $a_{i \sigma}^{+}$ 
and $n_{i \sigma}$. Unfortunately, the $b$-operators are not true fermion operators, because their commutation relations are

$$
\begin{aligned}
& {\left[b_{i \sigma}, b_{j}^{+} \tau\right]=\left(1-n_{i-\sigma}\right) \delta_{i j} \delta_{\sigma, \tau}+a_{j-\sigma}^{+} a_{j} \sigma \delta_{i j} \delta_{-\sigma, \tau},} \\
& {\left[b_{i \sigma}, b_{j} \tau\right]=\left[b_{i \sigma}^{+}, b_{j}^{+} \tau\right]=0 .}
\end{aligned}
$$

so that calculations with Eq. (2.1.3) are quite involved algebraically. We shall come back to this problem in the section devoted to the RVB model. One way to get rid of the inconvenient projected operators has been suggested by Spalek [26] in the spirit of the variational approach pioneered by Gutzwiller [27]. It has led to the mapping the Hubbard Hamiltonian in the large correlation limit $U>W$, via the projected Hamiltonian of Eqs. (2.1.1-2) above, onto the following effective Hamiltonian:

$$
\begin{aligned}
& H^{\mathrm{eff}}=\sum_{\langle i j\rangle, \sigma} \Phi_{\sigma} l_{i j} a_{i \sigma}^{+} a_{j \sigma}+U \sum_{i} n_{i \uparrow} n_{j \downarrow}+H^{\mathrm{Heeis}}, \\
& H^{\mathrm{Heis}} \equiv(1-\Phi)^{2} \sum_{\langle i, j\rangle} \frac{2 t_{i j}^{2}}{U}\left[S_{i} \cdot S_{j}-\frac{1}{4}\left(n-2 \eta_{i}\right)\left(n-2 \eta_{j}\right)\right],
\end{aligned}
$$

where we have introduced the probability of double occupancy of a given site by opposite spin electrons $\eta_{i} \equiv\left\langle n_{i \uparrow} n_{i \downarrow}\right\rangle$. It can be shown [26] that the expression of $\Phi$ in the PM phase, is $\Phi(n)=1-\left(1-f_{0}\right)\left(1-4 \eta / n^{2}\right)^{2}$ where $f_{0} \equiv(1-n) /(1-n / 2)$. One can show [26] that $\eta$ vanishes above a critical value of the ratio between correlation and bandwidth, whose evaluation depends on the shape of the DOS. For the rectangular DOS $U_{\text {crit }}=2 W$. When $\eta=0$, it follows $\Phi \rightarrow f_{0}$. Then the effective bandwidth of the system $W^{\text {eff }}=\Phi W \rightarrow f_{0} W \approx(1-n) W$, so that the presence of holes in the system is needed to have metallic properties. Due to the energy renormalization $\varepsilon_{k} \rightarrow \Phi \varepsilon_{k}$ also the DOS is modified according to $\rho_{\sigma}^{*}(E=\Phi \varepsilon)=(1 / \Phi) \rho_{\sigma}(\varepsilon)$. When $\Phi \rightarrow 0$, then $\rho^{*}$ diverges, indicating that the band reduces to a set of localized energy levels. At half-filling the bandwidth vanishes, turning the system into an insulator. The $t-J$ model is the limit for $U \gg W$ and $n \rightarrow 1$ of the $H^{\text {eff }}$ of Eq. (2.1.5). Looking at Eq. (2.1.6) one notices that the coefficient of the Heisenberg-like spin product has a coefficient, equivalent to an exchange interaction, intrinsically positive, implying a tendency of the system to order antiferromagnetically. In the next section we shall indeed study the AF state.

\subsection{The antiferromagnetic state in the strong correlation limit}

The first step will be a manipulation of $H^{\text {Heis }}$ to put it in a tractable form. Our starting point will be the remark that, in the subspace of states with no double occupancy of the lattice sites, the local moment $S_{i}^{z} \equiv(1 / 2)\left(n_{i \uparrow}-n_{i \downarrow}\right)$ reduces to $S_{i}^{z}=\sigma_{i} n_{i \sigma} / 2$ as either one of $n_{i \sigma}$ 's has to vanish. On the other hand, $\left\langle n_{i \sigma}\right\rangle=\left(n-2 \eta_{i}\right)$ so that

$$
\left\langle S_{i}^{z}\right\rangle\left\langle S_{j}^{z}\right\rangle=\frac{\sigma_{i} \sigma_{j}}{4}\left(n-2 \eta_{i}\right)\left(n-\eta_{j}\right)=-\frac{1}{4}\left(n-2 \eta_{i}\right)\left(n-\eta_{j}\right),
$$

because in the AF state $\sigma_{i} \sigma_{j}=-1$. Next let us introduce the identity

$$
S_{i} \cdot S_{j}=S_{i}^{z} S_{j}^{z}+\frac{1}{2}\left(S_{i}^{+} S_{j}^{-}+S_{i}^{-} S_{j}^{+}\right) \text {. }
$$


In the ordered state, the fluctuations of the order parameter are small, therefore we can safely use the IIartree-Fock condition $\left(\left\langle S_{i}^{z}\right\rangle-S_{i}^{z}\right)\left(\left\langle S_{i}^{z}\right\rangle-S_{j}^{z}\right) \approx 0$ yielding

$$
S_{i}^{z} S_{j}^{z} \approx S_{i}^{z}\left\langle S_{j}^{z}\right\rangle+\left\langle S_{i}^{z}\right\rangle S_{j}^{z}-\left\langle S_{i}^{z}\right\rangle\left\langle S_{j}^{z}\right\rangle .
$$

Consistently with the assumption of the $\Lambda \mathrm{F}$ order being firmly established, we shall drop altogether the contribution $\left(S_{i}^{+} S_{j}^{-}+S_{i}^{-} S_{j}^{+}\right)$to $S_{i} \cdot S_{j}$. By expressing $S^{z}$ in terms of $n_{\sigma}$, the AF part of the effective IIamiltonian due to the localized particles can be cast in the form

$$
H^{\mathrm{Ieis}} \equiv \frac{1}{2} \sum_{\langle i, j\rangle} J_{i j} \sum_{\sigma} \sigma\left(\left\langle S_{j}^{z}\right\rangle n_{i \sigma}+\left\langle S_{i}^{z}\right\rangle n_{j \sigma}\right) .
$$

We shall limit our description of the AF phases to the Néel state as we did in the low-correlation case. We shall consider the local moments to belong to two interpenetrating sublattices $A$ and $B$, such that the $z$ nearest neighbours $j$ of a given site $i$ of the $A$ sublattice, with moment $S_{i_{A}}^{z} \equiv S^{z}$, all belong to $B$ and have a moment $S_{j_{B}}^{z}=-S^{z}$. Each $j_{B}$ site is connected to an $i_{A}$ site by a vector $d$ independent of the site: $\boldsymbol{R}_{j}=\boldsymbol{R}_{\boldsymbol{i}}+\boldsymbol{d}$. We shall define a space-averaged exchange interaction according to

$$
J \equiv \frac{1}{z} \sum_{j(i)} J_{i j}=\frac{2(1-\Phi)^{2}}{U}\left[\frac{1}{z} \sum_{d} t_{i, i+d}^{2}\right]=(1-\Phi)^{2} \frac{2 \iota^{2}}{U}=\frac{(1-\Phi)^{2} W^{2}}{2 z^{2} U},
$$

where $W \equiv 2 z t$ with $t^{2}$ the average of the hopping matrix element squared. Now we can write the final form of $I^{\text {Heis }}$ in terms of reciprocal space vectors as

$$
\begin{aligned}
& \frac{H^{\mathrm{AF}}}{N}=\frac{1}{N} \sum_{p_{\sigma}}\left[\Phi \varepsilon_{p} n_{p_{\sigma}}+\Phi \varepsilon_{p+q} n_{p+q, \sigma}\right]+\frac{U}{N} \sum_{i} n_{i \uparrow} n_{i \downarrow} \\
& -\frac{z J S q}{2 N} \sum_{p_{\sigma}} \sigma\left(a_{p_{\sigma}}^{\dagger} a_{p+q, \sigma}+a_{p+q, \sigma}^{\dagger} a_{p_{\sigma}}\right) .
\end{aligned}
$$

The calculations now proceed exactly as in the low-correlation case, with $J$ playing the role of $U$ there, so that we leave them to the reader and give only the final result for the order parameter for the rectangular DOS

$$
S_{\boldsymbol{q}}^{z}=\left[\frac{y / W}{\operatorname{Sh}(y)}\right] \sqrt{\varepsilon_{\mathrm{F}}^{2}+\varepsilon_{\mathrm{L}}^{2}-2\left|\varepsilon_{\mathrm{F}} \varepsilon_{\mathrm{L}}\right| \mathrm{Ch}(y)}
$$

where $y \equiv 2 \Phi W / z J$. As we found in the case $U<W$, the moment decreases exponentially fast from the fully localized value $n / 2$ when the itineracy (here expressed by $\Phi W)$ is appreciable. The AF state of the HTCSC materials is still not completely understood, particularly in the non-stoichiometric composition but we cannot discuss the subject further. 


\subsection{BCS-like pairing by transverse spin fluctuations}

We want to show that the Hamiltonian of Eq. (2.1.5) contains the possibility of pairing particles with opposite spins to form a Cooper pair. The resulting superconducting state, even when described by the standard BCS equations $[28,29]$ has unusual properties, among which we shall consider only two: the critical temperature $T_{\mathrm{c}}$, which can be unusually high, and its variation with pressure $P$, i.e. $\mathrm{d} T_{\mathrm{c}} / \mathrm{d} P$, which can be large and positive. We shall follow with some modifications Ref. [29]. The starting point is the development of $S_{i} \cdot S_{j}=S_{i}^{z} S_{j}^{z}+$ $\left(S_{i}^{+} S_{j}^{-}+S_{j}^{+} S_{i}^{-}\right) / 2$ in the spin-1/2 fermion representation $S_{i}^{z} \equiv\left(n_{i \uparrow}-n_{i \downarrow}\right) / 2$, $S_{i}^{+} \equiv a_{i \uparrow}^{+} a_{i \downarrow}, S_{i}^{-} \equiv a_{i \downarrow}^{+} a_{i \uparrow}$, yielding

$$
\begin{aligned}
& H=\Phi \sum_{i \neq j, \sigma} t_{i j} a_{i \sigma}^{\dagger} a_{j \sigma}+U \sum_{i} n_{i \uparrow} n_{i \downarrow} \\
& +\frac{2(1-\Phi)^{2}}{U} \sum_{i \neq j} t_{i j}^{2}\left[S_{i}^{z} S_{j}^{z}+\frac{\left(a_{i \uparrow}^{\dagger} a_{i \downarrow} a_{j \downarrow}^{\dagger} a_{j \uparrow}+\text { II.c. }\right)}{2}-\frac{\nu_{i} \nu_{j}}{4}\right] .
\end{aligned}
$$

To proceed, we replace the IIubbard term $U \sum_{i} n_{i \uparrow} n_{i \downarrow}$ by $U N \eta$ according to the definition of $\eta$ above and we make a mean-field decoupling of the terms from $S_{i} \cdot S_{j}$. Namely, the non-diagonal four-operator terms will be approximated as follows:

$$
\begin{gathered}
a_{i \uparrow}^{\dagger} a_{i \downarrow} a_{j \downarrow}^{\dagger} a_{j \uparrow}=-a_{i \uparrow}^{\dagger} a_{j \downarrow}^{\dagger} a_{i \downarrow} a_{j \uparrow} \approx-a_{i \uparrow}^{\dagger} a_{j \downarrow}^{\dagger}\left\langle a_{i \downarrow} a_{j \uparrow}\right\rangle \\
-\left\langle a_{i \uparrow}^{\dagger} a_{j \downarrow}^{\dagger}\right\rangle a_{i \downarrow} a_{j \uparrow}+\left\langle a_{i \uparrow}^{\dagger} a_{j \downarrow}^{\dagger}\right\rangle\left\langle a_{i \downarrow} a_{j \uparrow}\right\rangle
\end{gathered}
$$

and similarly for the Hermitian conjugate. The minus sign introduced by commutating operators referring to different sites and spins is of fundamental importance, because it is responsible for transforming an apparently positive (i.e. particle repelling) interaction into a negative (i.e. particle attracting) one, therefore opening the way for the Cooper pair formation even in the absence of the attractive electron-phonon interaction.

We shall study the phase with no $\mathrm{AF}$ order so that $\left\langle S_{i}^{z}\right\rangle=0$ at all temperatures and for all values of $n$, including $n=1$. Of course, at the end of the calculations one has to check, for given $n$, whether or not the assumed phase is more stable than the AF phase. By introducing as usual the isotropic hopping approximation, one has $J=(1-\Phi)^{2}\left(2 t^{2} / U\right)=(1-\Phi)^{2}\left(W^{2} / 2 z^{2} U\right)$ where we use $W \equiv 2 z t$. We then obtain in the reçiprocal space representation a Ilamiltonian with a BCS-like term

$$
\begin{aligned}
H= & \Phi \sum_{k, \sigma} \varepsilon_{k_{k}} n_{k_{j} \sigma}+U N \eta-\frac{J}{2} \sum_{k}\left(a_{k_{\uparrow} \uparrow}^{+} a_{-k_{\downarrow} \downarrow}^{+} A_{k}+\text { II.c. }\right) \\
& -\frac{J N}{4}\left[(n-2 \eta)^{2}+2\left\langle a_{i \downarrow} a_{j \uparrow}\right\rangle\left\langle a_{i \downarrow} a_{j \uparrow}\right\rangle\right],
\end{aligned}
$$

where $N$ is the number of sites, $j$ is a nearest neighbour of $i$, the vectors $k$ belong to the Brillouin zone and $\varepsilon_{k}=z t \gamma_{k}$ with $\gamma_{k}=(1 / z) \sum_{\langle j i\rangle} \mathrm{e}^{\mathrm{i} k \cdot\left(\boldsymbol{R}_{i}-\boldsymbol{R}_{j}\right)}$.

The order parameter is now $A_{k}=z \gamma_{k}\left\langle a_{i \uparrow} a_{j \downarrow}\right\rangle$. It describes the coupling of electrons with opposite spins on nearest neighbour sites. This means that the 
corresponding Cooper pair is very localized in real space, and therefore it is a wave packet with many Fourier components. By comparison, the original BCS picture of phonon coupled Cooper pair yields a wave packet spread over hundreds of lattice cells, being by consequence very localized in the reciprocal space. In a synthetic way one can say that the SC coherence length and the range $\delta\{k\}$ of $k$-vectors present in the wave packet are linked by $\xi \approx 1 / \delta\{k\}$ so that the present, and the classical, models represent the two opposite limits of, respectively, very small $\xi$ and very small $\delta\{k\}$. To interpret Eq. (2.3.3) as a BCS-like IIamiltonian we identify the coefficient of the last term $J / 2 \equiv V$ as the analog of the electron-phonon averaged matrix element. The gap parameter $\Delta_{k} \equiv V A_{k}$ is assumed isotropic in the following, $\Delta_{k} \rightarrow \Delta$. In the normal state $(\Delta=0)$ the equilibrium value of $\eta$ at $T=0$ can be found by minimizing the ground state energy. To describe the DOS for given spin $\sigma$ of the uncorrelated electrons in the normal state we choose the rectangular shape, which yields $\eta$ vanishing for $U / W>2$. The normal state of the ITCSC constitutes a fascinating field of research and is, at the moment, rather poorly understood. We shall leave a concise discussion of the PM state in the RVB picture for the following section.

We shall now proceed to analyse the correlation-mediated (as opposed to phonon-mediated) superconducting state by using the classical formal structure of BCS theory [2]. Some care is needed when applying the BCS formalism to a strongly correlated electron liquid. We arbitrarily fix a cut-off pairing frequency $\omega$ (corresponding to the Debye frequency $\omega_{\mathrm{D}}$ in the case of phonon coupling) by $\hbar \omega=k_{\mathrm{B}} T_{\mathrm{N}}$, where $T_{\mathrm{N}}$ is the hypothetical Néel temperature for a material with the same effective exchange $J$. It can be shown [29] that the choice of $\omega$ is irrelevant to the general conclusions. The mean-field relation for the three-dimensional case $k_{\mathrm{B}} T_{\mathrm{N}}=2 z J S(S+1) / 3$ when $S=1 / 2$ yields $k_{\mathrm{B}} T_{\mathrm{N}}=z J / 2$. The BCS theory of the SC state [2] yields two bands $E_{k}^{\mp}=\mp \sqrt{\xi_{k}^{2}+\Delta_{k}^{2}}$ with $\xi \equiv \varepsilon_{k}-E_{\mathrm{F}}$, where $E_{\mathrm{F}}=\Phi W(n-1) / 2$ is the Fermi level. The bands are separated by a gap $2 \Delta_{k}$ and at zero temperature $\Delta_{k}$ obeys the equation $\Delta_{k}=\sum_{q} V_{k-q} \Delta_{q} / \sqrt{\xi_{q}^{2}+\Delta_{q}^{2}}$. By passing to the isotropic approximation for $V_{k}$ and $\Delta_{k}$ and to the integration over the energies, the gap equation reads

$$
\frac{2}{\rho_{\sigma}^{*}\left(E_{\mathrm{F}}\right) V}=2\left(\frac{2 \Phi W}{J}\right)=\int_{\xi_{1}}^{\xi_{\mathrm{u}}} \frac{\mathrm{d} \xi}{\sqrt{\Delta^{2}+\xi^{2}}}
$$

where $\xi_{\mathrm{u}}$ and $\xi_{1}$ are, respectively, the upper and lower boundary of the energy interval over which the coupling is effective. In the phonon mediated case, $\xi_{\mathrm{u}}=-\xi_{\mathrm{l}}=\hbar \omega_{\mathrm{D}}$, independent of any other feature (e.g. bandwidth or band filling) of the system, while the present case can be different. Let us consider the case of $U / W \approx 2$ so that $\Phi(n \approx 1) \ll 1$ and think of increasing $n$ at fixed $U / W$. When $\Phi(n)$ is large, $\hbar \omega<\left|\Phi(n) W / 2 \pm E_{\mathrm{F}}(n)\right|$ and $\xi_{\mathrm{u}}=-\xi_{\mathrm{l}}=\hbar \omega$, in analogy with the phonon case. But as $n$ grows we come to the value $n=n_{1}$ for which $\hbar \omega=\Phi\left(n_{1}\right) W / 2+E_{\mathrm{F}}\left(n_{1}\right)$. For $n>n_{1}$, while $\xi_{\mathrm{u}}=\hbar \omega$ as before, $\xi_{1}$ becomes independent of $\omega$ and dependent on $n: \xi_{1}=-\Phi(n) W n / 2$. As $n$ increases further, we arrive at $n=n_{\mathrm{u}}$ such that $\hbar \omega=\Phi\left(n_{\mathrm{u}}\right) W / 2-E_{\mathrm{F}}\left(n_{\mathrm{u}}\right)$. For $n>n_{\mathrm{u}}$ we have $\xi_{\mathrm{u}}=\Phi(n) W(2-n) / 2$ : both integration limits are independent of $\omega$, depending only on $n$ for given $W$ and $x$. It is important to note that in the small- $\Phi$ limit, 
when $n_{\mathrm{u}}<n \leq 1$, one expects a rather small a verage radius (or coherence lengtli) of the Cooper pairs, because the corresponding wave function includes $k$-vectors from the whole Brillouin zone implying a small spread in the real space of the corresponding wave packet. This is consistent with the small coherence length observed in the high- $T_{\mathrm{c}}$ materials. From Eq. (2.3.4) one finds

$$
\Delta=\frac{2 \Gamma \hbar}{\left(\Gamma^{4}-1\right)} \sqrt{\Gamma^{2}\left(\xi_{\mathrm{u}}^{2}+\xi_{\mathrm{l}}^{2}\right)-\xi_{\mathrm{u}} \xi_{\mathrm{l}}\left(1+\Gamma^{4}\right)},
$$

where $\Gamma \equiv \exp \left[1 / \rho_{\sigma}^{*}\left(E_{\mathrm{F}}\right) V\right]$. The equation for $T_{\mathrm{c}}$ is [2]:

$$
\frac{2}{V}=\int_{\xi_{1}}^{\xi_{\mathrm{u}}} \rho_{\sigma}^{*}(\xi) \mathrm{Th}\left(\frac{\xi}{2 k_{\mathrm{B}} T_{\mathrm{c}}}\right) \frac{\mathrm{d} \xi}{\xi} .
$$

Changing variables from $\xi$ to $\zeta \equiv \xi / \Phi$ and writing down explicitly $V$ and $\rho_{\sigma}^{*}$ yields

$$
\frac{2 \Phi W}{J}=\int_{\xi_{1} / \Phi}^{\xi_{\mathrm{u}} / \Phi} \mathrm{Th}\left[\frac{\zeta}{2 k_{\mathrm{B}}\left(T_{\mathrm{c}} / \Phi\right)}\right] \frac{\mathrm{d} \zeta}{2 \zeta} .
$$

To proceed we have to consider separately the different cases for the energy cut-off. This choice between different cut-offs has a physical origin analogous to the cut-offs in the spin bag model discussed previously. For $\Phi$ large, $\xi_{\mathrm{u}}=-\xi_{1}=\hbar \omega$ and $\zeta \Phi / k_{\mathrm{B}} T_{\mathrm{c}} \gg 1$ over most of the integration range because $\hbar \omega \gg k_{\mathrm{B}} T_{\mathrm{c}}$, so that one can resort to the usual BCS approximation $\mathrm{Th}\left[\Phi \zeta / 2 k_{\mathrm{B}} T_{\mathrm{c}}\right] \approx 1$ yielding

$$
\frac{2 \Phi W}{J}=\ln \left[\frac{1.14(\hbar \omega / \Phi)}{k_{\mathrm{B}}\left(T_{\mathrm{c}} / \Phi\right)}\right] .
$$

We obtain the analogous of the BCS formula: $k_{\mathrm{B}} T_{\mathrm{c}}=1.14 \hbar \omega \exp (-2 \Phi W / J)$. When $\Phi W \ll 2 \hbar \omega$ the approximation over which the derivation of Eq. (2.3.8) from Eq. (2.3.6) is based is no longer valid and one has to solve Eq. (2.3.6) numerically. An explicit solution can be obtained in the limit of vanishing $\Phi$, i.e. for $n \rightarrow 1$ and $U / W>2$. Indeed, in that case $\xi_{\mathrm{u}}=-\xi_{\mathrm{I}}=\Phi W / 2$ and $\Phi \zeta / 2 k_{\mathrm{B}} T_{\mathrm{c}} \ll 1$ so that $\operatorname{Th}\left(\Phi \zeta / 2 k_{\mathrm{B}} T_{\mathrm{c}}\right) \approx \Phi \zeta / 2 k_{\mathrm{B}} T_{\mathrm{c}}$. We obtain the following expression:

$$
k_{\mathrm{B}} T_{\mathrm{c}}=\frac{V}{4}=\frac{J}{8} .
$$

It can be verified at once that the result above is consistent with the assumption $\Phi \zeta / 2 k_{\mathrm{B}} T_{\mathrm{c}} \ll 1$ made in the derivation. We can also explicitly evaluate $\Delta / k_{\mathrm{B}} T_{\mathrm{c}}$ in the insulating limit. As $y \equiv 1 / \rho_{\sigma}^{*}\left(E_{\mathrm{F}}\right) V \ll 1$, we can develop $\Delta$ from Eq. (2.3.5) from $\Gamma \approx 1+y$ yielding

$$
2 \Delta \approx \frac{\Phi W}{2 y}=\frac{J}{4}=4 k_{\mathrm{B}} T_{\mathrm{c}} .
$$

Notice that the above equation sets a general upper boundary [30] to the increase in the $2 \Delta / k_{\mathrm{B}} T_{\mathrm{c}}$ ratio due to the enhancement of the DOS value at the Fermi level within the weak coupling approximation. In other words, independently of the specific coupling interaction, if the efficiency of coupling is low (i.e. the coupling is weak) that ratio cannot increase beyond the value 4 . This is relevant in connection with the much higher (and very controversial!) values reportedly observed in the HTCSC.

The BCS formalism correctly applied to the present model gives a non-vanishing $T_{\mathrm{c}}$ (actually, a maximum of $T_{\mathrm{c}}(n)$ for given $U / W$ ) at the half-filled band 
limit $[28,29,31]$. This is a non-physical feature, due to the use of a mean-field approximation. What is physically significant in the above calculations is that they show how purely electronic interactions can be the origin of superconductivity with critical temperatures determined by the magnetic $(J)$, and not by the vibrational $\left(\hbar \omega_{D}\right)$ energy scale. Of course, such a magnetic-pairing sustained SC phase is in competition with at least the AF phase, and the compresence of AF and SC phases in the phase diagram is expected. In connection with the IITCSC materials, it is interesting to note that such a mechanism of superconductivity would explain the observed increase in $T_{\mathrm{c}}$ under pressure, because $k_{\mathrm{B}} T_{\mathrm{c}} \approx J=2 t^{2} / U$ and $\ell$ increases under pressure, as already mentioned.

The mechanism discussed above is non-standard, being independent of the electron-phonon interaction, but, once the phonon-independent origin of the coupling is clarified, the development of the model follows the standard procedure for condensation of the Fermi liquids. We shall now briefly describe another model, the resonating valence bond (RVB) proposed by Anderson and co-workers which, starting from the same general framework of strongly correlated electron liquid, opens quite different directions of investigation.

\subsection{Superconducting stales in the fermion RVB picture}

The RVB model was invented by Anderson to study the magnetic triangular lattice [32] well before the discovery of IITCSC's. We shall discuss it under two points of view: first, by analysing the nature of the quasi-particles forming the condensed state introduced in the previous section. Second, we shall introduce the slave boson representation of the RVB model, which opens a quite new way of thinking about fermions in strongly correlated systems.

To study the nature of the quasi-particles in the SC state, we shall follow $[28,31-33]$ by starting from Eq. (2.3.3) above in the limit $U \gg W$ so that $\eta=0$ and $\Phi \rightarrow f_{0}$. Let us observe that the mean field approximation to the $S_{i}^{+} S_{j}^{-}+$II.c. term we employed in Eq. (2.3.2) was not the most general one. Actually one should write, e.g.

$$
\begin{aligned}
& a_{i \uparrow}^{\dagger} a_{i \downarrow} a_{j \downarrow}^{\dagger} a_{j \uparrow}=-a_{i \uparrow}^{\dagger} a_{j \downarrow}^{\dagger} a_{i \downarrow} a_{j \uparrow} \approx-a_{i \uparrow}^{\dagger} a_{j \downarrow}^{\dagger}\left\langle a_{i \downarrow} a_{j \uparrow}\right\rangle-\left\langle a_{i \uparrow}^{\dagger} a_{j \downarrow}^{\dagger}\right\rangle a_{i \downarrow} a_{j \uparrow} \\
& -a_{i \uparrow}^{\dagger} a_{j \uparrow}\left\langle a_{j \downarrow}^{\dagger} a_{i \downarrow}\right\rangle-\left\langle a_{i \uparrow}^{\dagger} a_{j \uparrow}\right\rangle a_{j \downarrow}^{\dagger} a_{i \downarrow}+\left\langle a_{i \uparrow}^{\dagger} a_{j \downarrow}^{\dagger}\right\rangle\left\langle a_{i \downarrow} a_{j \uparrow}\right\rangle+\left\langle a_{i \uparrow}^{\dagger} a_{j \uparrow}\right\rangle\left\langle a_{j \downarrow}^{\dagger} a_{i \downarrow}\right\rangle .
\end{aligned}
$$

The first two terms in the second line of (2.4.1) describe the coherent hopping process whereby two opposite spin particles exchange sites $i$ and $j$. Their amplitudes assumed isotropic and spin-independent, are $p \equiv(J / 2)\left\langle a_{i \sigma}^{\dagger} a_{j \sigma}\right\rangle$. Such a process is distinguished from the incoherent hopping of the kinetic energy term with amplitude $\varepsilon_{\boldsymbol{k}}$, where each particle moves independently. After applying the decoupling (2.4.1) we can Fourier transform the remaining two-operator terms. When working at arbitrary temperature with quasi-particles created through a Bogolyubov transformation, which does not preserve the number of quasi-particles, it is convenient to use the grand canonical ensemble. If $\mu(n, T)$ is the chemical potential, then the reciprocal space representation of the $t-J$ IIamiltonian within the mean field approximation (2.4.1) is [31]:

$$
H=\sum_{k_{\sigma}}\left(\varepsilon_{k}-\mu\right) a_{k_{\sigma} \sigma}^{\dagger} a_{k \sigma}-\frac{J}{2} \sum_{k}\left(\Delta \gamma_{k} a_{k_{\dagger}}^{\dagger} a_{-k_{\downarrow}}^{\dagger}+\text { II.c. }\right)-N\left(\Delta^{2}+p^{2}\right),
$$




$$
\gamma_{k} \equiv \cos \left(k_{x} a_{x}\right)+\cos \left(k_{y} a_{y}\right), \quad \varepsilon_{k} \equiv\left(f_{0} l+p\right) \gamma_{k} .
$$

Notice that the bandwidth $W=2 z\left(f_{0} t+p\right)$ has contributions from both coherent and incoherent hopping. In this sense one can say that the magnetic fluctuations, contributing the coherent hopping amplitude, enlarge the bandwidth. Now let us diagonalize the IIamiltonian by the transformation to quasi-particle operators $c_{1 k}, c_{2 k}$ :

$$
c_{1 k}^{\dagger} \equiv a_{k \uparrow}^{\dagger} \cos \theta_{k}-a_{-k \downarrow} \sin \theta_{k}, \quad c_{2 k}^{\dagger} \equiv a_{k \uparrow} \cos \theta_{k}+a_{-k \downarrow}^{\dagger} \sin \theta_{k} .
$$

The transformation creates quasi-particles composed by the states having opposite spins and wave vectors, i.e. those which are coupled in a singlet Cooper pair. Notice that even though the new operators are spin independent, nevertheless their commutation relations are fermion-like: $\left[c_{1 k}^{\dagger}, c_{1} p\right]_{+}=\delta_{k p p}$. The diagonalization condition on $\theta_{k}$ is

$$
\begin{aligned}
& \cos \left(2 \theta_{k}\right)=\frac{\left|\varepsilon_{k}-\mu\right|}{\sqrt{\left(\varepsilon_{k}-\mu\right)^{2}+\left(\gamma_{k} \Delta\right)^{2}}}, \\
& \sin \left(2 \theta_{k_{k}}\right)=\frac{\left|\gamma_{k_{k}}\right| \Delta \operatorname{sgn}\left(\varepsilon_{k}-\mu\right)}{\sqrt{\left(\varepsilon_{k}-\mu\right)^{2}+\left(\gamma_{k} \Delta\right)^{2}}},
\end{aligned}
$$

implying that $\sin \theta_{k}>(<) 0$ if $k>(<) k_{\mathrm{F}}$, where the Fermi wave vector $k_{\mathrm{F}}$ is defined by $\varepsilon_{k_{\mathrm{F}}}=\lim _{T \rightarrow 0} \mu(n, T)$. The $c_{k}$ 's operators describe the RVB quasi-particles, whose spectrum has unusual properties both in the normal and the superconducting state. Indeed, the diagonalized IIamiltonian has two bands of quasi-particles with energies

$$
E_{1 k}=E_{2 k}=\operatorname{sgn}\left(\varepsilon_{k}-\mu\right) \sqrt{\left(\varepsilon_{k}-\mu\right)^{2}+\left(\gamma_{k} \Delta\right)^{2}} .
$$

In the usual BCS treatment, one has that a pair of states $|k \sigma\rangle$ and $|-k,-\sigma\rangle$, with the same energy $\varepsilon_{k}$ are mapped onto two SC states with energies $\pm \sqrt{\left(\varepsilon_{k}-E_{\mathrm{F}}\right)^{2}+\Delta^{2}}$. Here the same pair is mapped onto a single, doubly degenerate, state with energies given by (2.4.5). Ilowever, according to the position of $k$ with respect to the Fermi surface, determining the sign of $\varepsilon_{k}-\mu$, such state can have one of two energies $\pm\left|E_{1 k}\right|$. One very important consequence is that, when $\varepsilon_{k} \rightarrow \mu$, i.e. on the FS, the two possibilities coalesce as $\lim _{k \rightarrow k_{\mathrm{F}}^{+}} E_{1 k}=$ $\lim _{k \rightarrow k_{\mathrm{F}}^{-}} E_{1 k}=\gamma_{k_{\mathrm{F}}} \Delta$. Namely, the gap does not open for wave vectors on the FS. This should be reflected in, e.g. a linear rise of the specific heat with temperature $\left(C_{V} \approx T\right.$ instead of $C_{V} \approx \exp \left(-\Delta / k_{\mathrm{B}} T\right)$ as predicted by BCS [2]) as well as in modifications of other experimental observables [31, 34].

The SC state of the transformed IIamiltonian has been studied essentially by the techniques described in the preceding section in Refs. [31, 35]. We shall not repeat their calculations because they do not add new concepts. The distinction between the SC and the normal (quite unusual, actually) RVB states can be made only after self consistently solving the equations for the critical temperature, the chemical potential and the coherent hopping amplitude. For some values of the parameters $J$ and $f_{0} \approx(1-n)$ there will be a stable SC phase. We shall not deal with those results for lack of space. The reader interested in the complete phase 
diagram of the RVB model is referred to $[28,31,34,36]$. Instead, we shall comment on the properties of the non-SC state, i.e. the state where both $\Delta$ and $p$ can be non-vanishing, without a long-range order.

Due to their fermion-like commutation properties, and to the absence of a gap at the Fermi surface (FS), just like in a normal metal, we expect that the quasi-particles described by Eq. (2.4.3) possess Fermi liquid (FL) properties, at least for $n<1$. Let us check if the FS really exists by evaluating the discontinuity $Z_{1 k_{\mathrm{F}}}$ in the occupation numbers for arbitrary $n$. In the usual FL this is evaluated per spin direction, while here each type 1,2 of quasi-particle mixes both spins. We shall consider the sum $Z_{k_{\mathrm{F}}} \equiv\left(Z_{1 k_{\mathrm{F}}}+Z_{2} k_{\mathrm{F}}\right) / 2$. We obtain from the transformation relations

$$
\begin{aligned}
& c_{1 k}^{\dagger} c_{1 k}+c_{2 k}^{\dagger} c_{2 k}=\left(n_{k \uparrow}+n_{-k \downarrow}\right) \cos ^{2} \theta_{k}+\left(2-n_{k \uparrow}-n_{-k_{\downarrow} \downarrow}\right) \sin ^{2} \theta_{k} \\
& -\left(a_{k \uparrow}^{\dagger} a_{-k \downarrow}^{\dagger}-a_{k \uparrow} a_{-k \downarrow}\right) \sin \left(20_{k}\right) .
\end{aligned}
$$

For $|k \sigma\rangle$ states inside the FS $\left\langle n_{k_{0} \sigma}\right\rangle=1$ while $\left\langle a_{k_{\dagger} \dagger}^{\dagger} a_{-k_{l}}^{\dagger}\right\rangle=0$ while the converse happens outside the FS. By taking into account the sign change of $\sin \theta_{k}$ on crossing the FS, one obtains

$$
\begin{aligned}
& Z_{k_{\mathrm{F}}} \equiv\left\langle c_{1 k^{\prime}}^{\dagger} c_{1 k}+c_{2 k^{\prime}}^{\dagger} c_{2 k_{i}}\right\rangle_{k \rightarrow k_{\mathrm{F}}^{-}}-\left\langle c_{1 k_{1}}^{\dagger} c_{1 k}+c_{2 k_{2 k}}^{\dagger} c_{2 k}\right\rangle_{k \rightarrow k_{F}^{+}}, \\
& z_{k_{\mathrm{F}}}=\frac{2\left|\varepsilon_{k}-\mu\right|_{k \rightarrow k_{\mathrm{F}}}-\left|\gamma_{k_{\mathrm{F}}} \Delta\right|\left[\left\langle a_{k_{\uparrow} \uparrow} a_{-k_{\downarrow} \downarrow}\right\rangle_{k_{\mathrm{F}}^{-}}-\left\langle a_{k_{\uparrow}}^{\dagger} a_{-k_{\uparrow}}^{\dagger}\right\rangle_{k_{\mathrm{F}}^{+}}\right]}{\sqrt{\left(\varepsilon_{k}-\mu\right)_{k-k_{\mathrm{F}}}^{2}+\left(\gamma_{k} \Delta\right)^{2}}} .
\end{aligned}
$$

If $\Delta \rightarrow 0$, then $Z_{k_{F}} / 2 \rightarrow 1$ and we recover the usual Fermi liquid result. But if $\Delta \neq 0$, for $k$ such that $\varepsilon_{k}=\mu$, presumably defining the Fermi wave vector $k_{\mathrm{F}}$, $Z_{k_{\mathrm{F}}}$ would be negative, which is unacceptable. Actually, following the Luttinger theorem [35], the Fermi wave vectors to consider are those determining the FS of the unperturbed system, i.e. when $J=0$ and by consequence $p=\Delta=0$. If $k_{F}$ is therefore redefined in such a way, and indicated by $k_{F}^{0}$, then at $k_{F}^{0}$ one has $1>Z_{k_{\mathrm{F}}^{0}} / 2 \geq 0$ where the vanishing requires

$$
\left|\varepsilon_{k}-\mu\right|_{k \rightarrow k_{\mathrm{F}}^{0}}=p=\left|\gamma_{k_{\mathrm{F}}^{0}} \Delta / 2\right|\left[\left\langle a_{k_{\uparrow} \uparrow} a_{-k_{\downarrow}}\right\rangle_{k_{\mathrm{F}}^{0-}}-\left\langle a_{k_{\uparrow} \uparrow}^{\dagger} a_{-k_{\downarrow}}^{\dagger}\right\rangle_{k_{\mathrm{F}}^{0+}}\right] \text {. }
$$

The picture of the RVB normal state is that of fermionic particles with some bosonic character (being composed of both up and down spins), having a gapless spectrum as in a usual metal, but with a possibly vanishing discontinuity in correspondence to the unperturbed FS. The non-zero value of $p$ indicates that the coherent fermion hopping is present in such a state together with some bosonic-like pair correlation expressed by $\Delta$. The Luttinger theorem [35] is violated, indicating that we are describing something different from a Fermi liquid. Indeed, such a state of fermionic quasi-particles with vanishing discontinuity at the FS is called the Luttinger liquid [33]. It is typical of systems where the spin and charge degrees of freedom are separated by a huge energy. Of its many interesting properties, we shall just mention one [31]. Let us see low the excitations of pairs of particles look like. We shall distinguish three cases, according to the wave vectors of the 
creation operators being both below, or one below and one above, or both above, the unperturbed FS. One has for arbitrary vectors $p, k$ :

$$
\begin{aligned}
& c_{1 k}^{\dagger} c_{1 p}^{\dagger}=a_{k \uparrow \uparrow}^{\dagger} a_{p \uparrow}^{\dagger} \cos \theta_{k} \cos \theta_{p}-a_{k \uparrow}^{\dagger} a_{-} p_{\downarrow} \cos \theta_{k} \sin \theta_{p} \\
& -a_{-k \downarrow} a_{p \uparrow}^{\dagger} \sin \theta_{k} \cos \theta_{p}+a_{-k_{\downarrow}} a_{-p \downarrow} \sin \theta_{k} \sin \theta_{p} .
\end{aligned}
$$

In the case $k, p<k_{F}$ the only relevant term is $a_{-k \downarrow} a_{-p \downarrow} \sin \theta_{k} \sin \theta_{p}\left(1-\delta_{k p}\right)$ which describes the creation of two holes (charged excitation) having the same down spin. If $p \leq k_{\mathrm{F}}<k$, we are left with $a_{k \uparrow}^{\dagger} a_{-} p_{\downarrow} \cos \theta_{k} \sin \theta_{p}$ describing a spin flip (spin excitation) with no change of the total charge. We can therefore create separately spin or charge excitations. This formal feature has a physical counterpart in the different scales of energy which correspond to perturbing, respectively, the magnetic configuration by flipping a spin $\left(J \approx W^{2} / U\right)$ and the charge distribution by creating a double occupancy $(U \gg J)$. The former excitation can be easily created, the latter cannot. This situation should be contrasted with the situation encountered when dealing with the spin bag model in the previous section, where it was basic to the argument that doping a hole into the system implied a change both in the charge and in the spin.

\subsection{Holon-spinon representation of the normal RVB state}

A very innovalive point of view has been subsequently developed in a series of papers by Anderson et al. [37-39]. Its roots are in one of the early papers by IIubbard [40] who suggested to deal with the strong correlation limit by considering the local term $U \sum_{i} n_{i \uparrow} n_{i \downarrow}$ as the unperturbed IIamiltonian and to treat the hopping term as a perturbation. The IIIlbert space of the local Ilamiltonian is composed by four states per each site. Each state $|\alpha\rangle$ corresponds to a given occupancy of the site $\{|\alpha\rangle\}=\{|0\rangle,|\uparrow\rangle,|\downarrow\rangle,|\uparrow \downarrow\rangle\}$. The state $|\uparrow \downarrow\rangle$ will also be indicated as $|2\rangle$ in the following. The local operators introduced by Ilubbard are $X_{i}^{\alpha \beta} \equiv|\alpha\rangle\langle\beta|$. In the following, the site label will be inserted only when needed for clarity. The diagonal operators $X_{i}^{\alpha \alpha}$ are projectors, obeying, for each site, the completeness relation

$$
\sum_{|\alpha\rangle} X_{i}^{\alpha \alpha}=1
$$

The $X^{\alpha \beta}$ operators allow one to use the diagrammatic techniques, as shown in Ref. [41]. Their commutation relations are

$$
\left[X_{i}^{\alpha \beta}, X_{j}^{\gamma \lambda}\right]_{ \pm}=\delta_{i j}\left(\delta_{\beta \gamma} X_{i}^{\alpha \lambda} \pm \delta_{\alpha \lambda} X_{i}^{\gamma \beta}\right),
$$

where the sign inside the commutator is + (Fermi-like) but in the case when at least one of the $X_{i}^{\alpha \beta}$ involved is Bose-like, i.e. produces an integer change of spin: $X_{i}^{00}, X_{i}^{\sigma \sigma}, X_{i}^{\sigma-\sigma}, X_{i}^{-\sigma \sigma}$. The usual Fermi operators $a_{i \sigma}, a_{i \sigma}^{\dagger}$ are expressed through the $X_{i}^{\alpha \beta}$ operators by considering all the combinations of the latter ones which yield the same effect as the application of the former ones, e.g. $a_{i \sigma}^{\dagger}|0\rangle=|\sigma\rangle$ etc. Then

$$
a_{i \sigma}=|0\rangle\langle\sigma|+\sigma|-\sigma\rangle\langle\uparrow \downarrow|=X_{i}^{0 \sigma}+\sigma X_{i}^{-\sigma 2},
$$




$$
a_{i \sigma}^{\dagger}=|\sigma\rangle\langle 0|+\sigma| \uparrow \downarrow\rangle\langle-\sigma|=X_{i}^{0 \sigma}+\sigma X_{i}^{2-\sigma} .
$$

The factor $\sigma= \pm 1$ is needed for the decomposed form of the $a_{i \sigma}$ operators to preserve the proper commutation properties. The $X^{\alpha \beta}$ operators share the properties of both Bose and Fermi operators. Their twofold nature can be made explicit by writing them in terms of purely fermionic $\left(f_{i \sigma}^{\dagger}\right)$ and purely bosonic $\left(b_{i}^{\dagger}, d_{i}^{\dagger}\right)$ operators [37] as follows:

$$
\begin{aligned}
& X_{i}^{00}=b_{i}^{\dagger} b_{i}, \quad X_{i}^{0 \sigma}=b_{i}^{\dagger} f_{i \sigma}, \quad X_{i}^{\sigma 0}=f_{i \sigma}^{\dagger} b_{i}, \quad X_{i}^{2 \sigma}=d_{i}^{\dagger} f_{i-\sigma}^{\dagger}, \\
& X_{i}^{\sigma 2}=f_{i-\sigma} d_{i}, \quad X_{i}^{\sigma \sigma}=f_{i \sigma}^{\dagger} f_{i \sigma}, \quad X_{i}^{22}=d_{i}^{\dagger} d_{i} .
\end{aligned}
$$

From these relations it is apparent that $b_{i}^{\dagger} b_{i}$ and $d_{i}^{\dagger} d_{i}$ are the number operators for, respectively, the empty and the doubly occupied sites. According to Eq. (2.5.3) the original Fermi operators now read

$$
a_{i \sigma}=b_{i}^{\dagger} f_{i \sigma}+\sigma d_{i} f_{i-\sigma}^{\dagger}, \quad a_{i \sigma}^{\dagger}=b_{i} f_{i \sigma}^{\dagger}+\sigma d_{i}^{\dagger} f_{i-\sigma} .
$$

The $k$-space representation of the $a_{i \sigma}$ operators is

$$
a_{k_{\sigma} \sigma}=b_{i}^{\dagger} f_{k \sigma}+\sigma d_{i} f_{k_{-\sigma}}^{\dagger}, \quad a_{k_{\sigma} \sigma}^{\dagger}=b_{i} f_{k_{\sigma} \sigma}^{\dagger}+\sigma d_{i}^{\dagger} f_{k-\sigma},
$$

where the site label on the boson part indicates that all Fourier components of the Bose operators in e.g. $b_{i} \equiv N^{-1 / 2} \sum_{q} b_{q} \exp \left(-i q \cdot \boldsymbol{R}_{i}\right)$ interact with each single component of the Fermi operators. As the set of the new operators is overcomplete, we need some constraint to get sensible results. It is provided by the obvious requirement that the original operators in the new form still retain their Fermi nature, i.e. $\left[a_{i \sigma}, a_{j \tau}^{\dagger}\right]_{+}=\delta_{\sigma \tau} \delta_{i j}$. If we evaluate the anticommutator for $i=j$ by using Eq. (2.5.5), we obtain that the decomposition preserves the statistics provided

$$
b_{i}^{\dagger} b_{i}+d_{i}^{\dagger} d_{i}+\sum_{\sigma} f_{i \sigma}^{\dagger} f_{i \sigma}=1
$$

It is easy to check that the equation above is obtained also from the completeness relation (2.5.1) for the $X$ operators when expressed through the new operators. Physically the constraint means that each site cannot be occupied by more that one particle, independently of its type. While usual bosons can occupy any state in arbitrary number, the $b_{i}$ and $d_{i}$ bosons have to obey the restriction above: their freedom is restricted, i.e. they are slave bosons. This has consequences on their statistics. Indeed, from the constraint it follows that the motions of the bosons and the fermion components of the clectron are correlated. Indeed, the flow $\phi^{x}$ of particles of generic type $x_{i \sigma}$ is non-vanishing if their number is not conserved locally. It therefore is given by the value of the commutator of the number operator $n_{i}^{x} \equiv \sum_{i \sigma} x_{i \sigma}^{\dagger} x_{i \sigma}$ with the IIamiltonian. From (2.5.6) it follows $\left[H, n_{i}^{b}\right]+\left[H, n_{i}^{d}\right]+\sum_{\sigma}\left[H, n_{i \sigma}^{j}\right]=[H, 1]=0$ or

$$
\phi^{b}+\phi^{d}=-\phi^{f} \text {. }
$$

As the $f$ particles move there has to be a flow in the opposite direction (a backflow) of bosons in order to fulfil the constraint. This means that as soon as a real electron is added to the system, it decomposes into two quasi-particles which travel 
oppositely! The fermion part will have to fulfil the Pauli principle, and therefore its momentum will be at least equal to the Fermi momentum, i.e. non-vanishing. The boson counterpart will be forced to move also with a non-vanishing momentum opposite to the former, while it would, if free, condense into the zero-momentum, lowest energy, state. Being enslaved by the constraint, it is obliged to move, hence to stay out of the condensed state. Baskaran, who discusses in more detail the process [42] has termed it transfer of stalistics. Its implementation into the mathematical description of the slave boson pairing will be dealt with in the next section.

The representation of the original operators in terms of slave bosons given above is, actually, too simple, and has some problems which we shall not discuss here. The reader interested in the more advanced developments is referred to [43].

Even if their use is more convenient in the strongly correlated limit, the slave boson technique is general. One can indeed write the Hubbard Hamiltonian for arbitrary $U$ in the new representation as follows:

$$
\begin{aligned}
& H=\sum_{\langle i j\rangle \sigma} t a_{i \sigma}^{\dagger} a_{j \sigma}+\frac{U}{2} \sum_{i \sigma} n_{i \sigma} n_{i-\sigma}-\mu \sum_{i \sigma} a_{i \sigma}^{\dagger} a_{i \sigma} \Rightarrow H_{0}+H_{1}, \\
& H_{0} \equiv \sum_{i j \sigma} t\left(b_{i} b_{j}^{\dagger}-d_{i} d_{j}^{\dagger}\right) f_{i \sigma}^{\dagger} f_{j \sigma}+\mu\left[\sum_{i}\left(b_{i}^{\dagger} b_{i}-d_{i}^{\dagger} d_{i}\right)-N\right]+U \sum_{i} d_{i}^{\dagger} d_{i} \\
& H_{1} \equiv t \sum_{\langle i j\rangle}\left[\left(b_{i} d_{j}+b_{j} d_{i}\right) f_{i \sigma}^{\dagger} f_{j-\sigma}^{\dagger}+\text { II.c. }\right] .
\end{aligned}
$$

The decomposition of the electron operators into bosonic and fermionic components yields an explicit separation of charge and magnetic degrees of freedom, which, as discussed previously in connection with the very different energy scales of the two types of excitations, is a desirable property for any representation of the strongly correlated systems. Following [37], let us observe that the boson operators $b_{i}^{\dagger}$ and $d_{i}^{\dagger}$ create excitations with charge $Q_{b}=-Q_{d}=+|e|$. Now, on each site, the number operator of the physical electrons, when decomposed according to Eq. (2.5.5) reads

$$
\sum_{\sigma} a_{i \sigma}^{\dagger} a_{i \sigma}=-b_{i}^{\dagger} b_{i}+d_{i}^{\dagger} d_{i}+1
$$

Given a generic particle $x_{i}=\left\{a_{i}, b_{i}, d_{i}\right\}$ the relative current follows from

$$
J_{x} \equiv \partial P_{x} / \partial t=\mathrm{i}\left[H, P_{x}\right], \quad P_{x} \equiv Q_{x} \sum_{i \sigma} R_{i} x_{i \sigma}^{\dagger} x_{i \sigma},
$$

where $P_{x}$ is the particle polarization operator. From Eq. (2.5.9) one has

$$
\left[H, Q_{a} \sum_{i \sigma} a_{i \sigma}^{\dagger} a_{i \sigma}\right]=\left[H, Q_{b} \sum_{i} b_{i}^{\dagger} b_{i}\right]+\left|Q_{d}\right|\left[H, \sum_{i} d_{i}^{\dagger} d_{i}\right],
$$

so that $J_{a}=J_{b}+J_{d}$, showing the electron current is completely accounted for by the spin-independent bosonic current. By consequence, the fermions $f_{i}$ do not carry charge. We conclude therefore that the slave bosons carry charge, but no spin, while the fermions carry spin but no charge. The pictorial view of the situation is that, if on a site there is one electron whose spin is not coupled in a singlet state to any other spin (not necessarily a nearest neighbour), then the spin pattern is 
disturbed, but the charge pattern is not. This configuration is the effect of applying $f_{i \sigma}^{\dagger}$ on the RVB ground state, where all spins are coupled in singlets with bonds of any length: Anderson termed such excitation of the RVB spin pattern a spinon. Spinons are always created in pairs, because both ends of a resonating bond are simultaneously broken. Once created, each spinon can move independently of its twin. Conversely, if one creates a hole or a double occupancy, the charge pattern is modified, while the spin pattern is not. Indeed, the spin on the site is zero, as the two true fermions have opposite spins, so that there is no unpaired spin, i.e. no spin excitation. The usually accepted name of the excitation creating a double hole is holon while some authors call the creation of a doubly occupied site a doublon. So far, the value of $U$ is arbitrary. To go to the case of large correlation, one performs [37] on the decomposed IIamiltonian (2.5.8) the same type of canonical transformation which yielded the kinetic exchange (or $t-J$ IIamiltonian). In the subspace without doubly occupied sites, $\left\langle d_{i}^{\dagger} d_{i}\right\rangle=0$ and we are left with:

$$
\begin{aligned}
H^{\mathrm{eff}} & =\sum_{\langle i j \sigma\rangle} t\left(b_{i} b_{j}^{\dagger} f_{i \sigma}^{\dagger} f_{j \sigma}+\text { II.c. }\right) \\
& +\mu\left(\sum_{i} b_{i}^{\dagger} b_{i}-N\right)-\frac{t^{2}}{U} \sum_{\langle i j\rangle}\left(f_{i \uparrow}^{\dagger} f_{j \downarrow}^{\dagger} f_{j \downarrow} f_{i \uparrow}+\text { II.c. }\right) .
\end{aligned}
$$

Notice that in Ref. [37] the coefficient of the last term is $4 t^{2} / U$ due to their use of the kinetic term with the IIermitian conjugate contribution written explicitly. The projected IIamiltonian has the charge carrying particles (bosons) appearing only in the kinetic term, while the exchange contribution involves only spinons. At this point one makes a mean-field approximation on the bosonic part, followed by an isotropic approximation by assuming $b_{i}^{\dagger} \rightarrow\left\langle b_{i}^{\dagger}\right\rangle \rightarrow\langle b\rangle$. It follows that the effective number of $b$ bosons is connected to the number of electrons in the band by

$$
\frac{\partial H}{\partial \mu} \equiv-N_{\text {electrons }}=b^{2} N-N,
$$

implying $b^{2}=\left(N-N_{\text {electrons }}\right) / N \equiv n_{\text {tioles }} /$ site. For the half-filled band case $\left(N_{\text {electrons }}=N\right)$ there are only virtual charge fluctuations as $\langle b\rangle^{2}=0$. The charge excitations become observable if some holes are doped into the system. From the constraint (2.5.6) it also follows that the spinon number $\sum_{\sigma} f_{i \sigma}^{\dagger} f_{i \sigma}=1-\langle b\rangle^{2}=n$.

To make a connection with the previous treatment of the RVB model in terms of configurations of true electrons, let us consider each term of the transformed Hamiltonian. The fermionic factor in the hopping term can be interpreted as describing the process by which a lone spin (a spinon) is destroyed on site $j$ and created on site $i$. This process by itself does not correspond to a real electron hopping, which additionally requires (see Eq. (2.5.5)) the concurrent presence of the bosonic process, i.e. the creation of a holon at $j$ and its annihilation at $i$. In a mean-field approximation for the bosonic process, the amplitude of the hopping is $t^{\mathrm{eff}}=t\langle b\rangle^{2}$. This result can be compared with Eq. (2.4.2), $t^{\mathrm{eft}}=f_{0} t \approx 2 t\langle b\rangle^{2}$ as $f_{0}=(1-n) /(1-n / 2) \approx 2(1-n)=2\langle b\rangle^{2}$. This shows that the two approaches, while giving essentially coinciding result for $n \rightarrow 1$, are not strictly identical when the number of holes is appreciable. This is not surprising, as we are considering an exactly transformed IIamiltonian but we are truncating its perturbative 
development. The different approaches might well yield coincident results if the perturbative development was carried on to infinite order, but there is no reason to expect equal order terms in the development to exactly coincide. The above analysis of the hopping term indicates in which sense one can say that a true electron is composed by a spinon and a holon. The two components may, under appropriate circumstances, have a different dynamics (however subject to the constraint (2.5.6)) which does not correspond to the motion of a true electron in the usual sense. This is apparent in the last term, where only the spinon operators appear.

The relation $\langle b\rangle^{2}=1-n$ implies that if the band is half-filled, there are no holons, and the transformed Ilamiltonian reduces to the last term, which has the same structure as the spin fluctuation term in (2.3.1). One can diagonalize it [37] by the same transformation, with an important difference. In the former case, we transformed physical particle operators (the electrons) whose number was a constant of the system in all its states. Then the quasi-particle spectrum (2.4.5) depended on the true chemical potential $\mu$ of the original particles. Here we have to transform operators $f_{i \sigma}$ not corresponding to physical particles, and whose number is not conserved. By consequence, there is no chemical potential for the $f_{i \sigma}$. This does not mean that we have no constraint on the overall number of quasi-particles. Indeed, in all states of the system, the constraint (2.5.6) has to be fulfilled. This is done by introducing a Lagrange multiplier for each site (in principle), which plays the role of a kind of chemical potential for the mixture of bosons and fermions. The chemical potential $\mu$ of the original IIamiltonian, does not depend on the site index being by definition the same everywhere in the crystal. Also, as $\mu$ is connected with the electrically charged physical particles, and the charge is carried only by the bosons, only boson operators multiply $\mu$ in (2.5.12). It follows that any diagonalization of (2.5.12) which involves the replacement of the Bose operators by their average values, yiclds a $\mu$-independent quasi-particle spectrum. For $n=1$ (so that $\langle b\rangle^{2}=0$ ) if one defines the RVB order parameter $\Delta \equiv\left\langle f_{i \sigma}^{\dagger} f_{j-\sigma}^{\dagger}-f_{j \sigma}^{\dagger} f_{i-\sigma}^{\dagger}\right\rangle$, then, in analogy with the limit $\varepsilon_{k} \rightarrow 0$ of (2.4.5), the quasi-particle spectrum reads: $E_{k_{\sigma}}^{f}=J \Delta\left|\gamma_{k}\right|$. If $n<1$ so that also the first term in (2.5.12) contributes, one has $E_{k}^{f}=\left|\gamma_{k}\right| \sqrt{l^{2}\langle b\rangle^{2}+(J \Delta)^{2}}$. The $f_{i \sigma}$ quasi-particles are gapless therefore and their bandwidth, for $n \approx 1$ is of the order of $\Delta J$.

To discuss briefly the dynamics of the coupled spinon-holon system, let us pass to the reciprocal lattice representation of the transformed IIamiltonian. The hopping term reads: $H^{\text {hop }}=t \sum_{k p q \sigma} b_{k} b_{p}^{\dagger} f_{q \sigma}^{\dagger} f_{p+q-k, \sigma} z \gamma_{q-k}$ which we separate into parts, i.e. with or without cliange in the boson wave vector, yielding

$$
H^{\text {hop }}=t \sum_{k} b_{k} b_{k}^{\dagger} \sum_{q \sigma} n_{q_{\sigma}}^{J} z \gamma_{q-k}+t \sum_{k \neq p, q_{\sigma}} b_{k} b_{p}^{\dagger} f_{q_{\sigma}}^{\dagger} f_{p+q-k, \sigma} z \gamma_{q-k},
$$

where $n_{q \sigma}^{f} \equiv f_{q \sigma}^{\dagger} f_{q_{\sigma} \sigma}$. The first contribution, diagonal in the wave vectors, describes the coherent motion of holons and spinons. If there we approximate in mean ficld the fermionic part to $H^{\text {hop }}$ and define $\left\langle\sum_{q \sigma} n_{q \sigma}^{f} \gamma_{q-k}\right\rangle \equiv \psi_{f}$, we have

$$
H^{\text {hop }}=t z \psi_{f} \sum_{k} b_{k} b_{k}^{\dagger}+t \sum_{k \neq p, q \sigma} b_{k} b_{p}^{\dagger} f_{q \sigma}^{\dagger} f_{p+q-k, \sigma} z \gamma_{q-k} .
$$


The first contribution describes the holon motion whose bandwidth $B=z l \psi_{f}$ depends on the spinon number $n_{f}$. As already required by the relation (2.5.7) between holon and spinon currents, the holon can move only if there are spinons in the system. Also, the mass of the holon is of the order of the electron mass because from (2.5.6) one has $\sum_{\sigma} n_{i \sigma}^{f}=n \approx 1$, implying $B \approx z l=W / 2$. Alternatively one can stress the itineracy of the spinons by writing

$$
H^{\text {hop }}=\sum_{\boldsymbol{q}_{\sigma}} t z n_{\boldsymbol{q} \sigma}^{f} \sum_{\boldsymbol{k}} b_{k} b_{k}^{\dagger} \gamma_{q-k}+\sum_{k \neq p, q_{\sigma}} b_{k} b_{p}^{\dagger} f_{q \sigma}^{\dagger} f_{p+q-k, \sigma} z \gamma_{q-k}
$$

By approximating $\sum_{k} b_{k} b_{k}^{\dagger} \gamma_{q-k} \approx\langle\zeta\rangle^{2}$ one obtains

$$
H^{\text {hop }}=z t\langle b\rangle^{2} \sum_{q_{\sigma}} n_{q_{\sigma}}^{f}+\sum_{k \neq p, q_{\sigma}} b_{k} b_{p}^{\dagger} f_{q \sigma}^{\dagger} f_{p+q-k, \sigma} z \gamma_{q-k} .
$$

showing the electron bandwidth renormalization due to the bosons. The part of the above IIamiltonian with a change in the boson wave vector describes the scattering of spinons and bosons, which has an amplitude of the order of the hopping bandwidth. When a real electron moves individually, i.e. in the normal state, its two components, the spinon and the holon, are scattered against the analogous components of other electrons and this appears as the electron-electron scattering contribution to the resistivity [37-39]. However, its temperature dependence, which goes as $T^{2}$ in usual Fermi liquids, is now expected to go as $T$. Indeed, it is proportional to the thickness $\approx k_{\mathrm{B}} T$ of spinon states around their Fermi surface times the distribution function of the final states. Now, in the absence of exclusion principles on the final holon states characteristic of bosons, the number of available final states is essentially determined (at $k_{\mathrm{B}} T \ll U$ ) by the hole doping due to the chemical composition, which is independent of temperature and fixes $\langle b\rangle^{2}=1-n$. Also, as $T \rightarrow 0$ the resistivity should vanish in samples of good purity, because the impurities are not decomposable into holons and spinons, so that they cannot scatter the composite electrons.

Now, let us schematize the IITCSC's as composed of conducting layers separated by insulating material, following [39]. At ligh temperatures, the interplane conductivity is due to the tunnelling of true clectrons between planes. Both components of the true electron have to jump simultaneously. On the starting plane, the electron is decomposed. A spinon moves out of the plane towards a neighbouring one, and its bosonic counterpart gocs the opposite way. If another electron (not necessarily on the same layer) decomposes into a holon landing on the adjacent plane together with the spinon, a single true electron has been transferred. Now the spinon, being a fermion, has to find a free state in the final layer. On each layer, the spinons form a band of width $\approx \Delta J$ as shown before, hence the spinon Fermi level will have a value of the same order. The incoming spinon has to overcome a barrier $\approx J$ having as propeller the thermal energy $\approx k_{\mathrm{B}} T$. Hence its jumping probability will go as $k_{\mathrm{B}} T / J$ and the perpendicular resistivity as $J / k_{\mathrm{B}} T$. The RVB model in the slave boson version, therefore, yields [39] a brilliant explanation of the reportedly quite different behaviour of the in-plane and out-of-plane resistivity, the former being linear in $T$, more or less as in a metal, the latter going as $T^{-1}$ reminiscent of an insulator. What happens at $T=T_{\mathrm{c}}$ in this picture? Well, 
$T_{\mathrm{c}}$ is just the lower temperature supplying enough energy to the spinon to allow it to overcome the Fermi level in the adjacent layers and to follow its bosonic counterpart in transferring a true electron between planes. Below $T_{\mathrm{c}}$ this is not possible. Notice that in this model $T_{\mathrm{c}}$ grows with $J$ and with the interlayer spacing, because the width of the insulating layer is a further obstacle to the passage of the spinon. At temperatures low enough to confine the spinons inside the layers, only the holons can move between layers.

The superconducting state according to the RVB model is due to pair holon condensation. A thorough discussion would require introducing the paired boson problem, which is quite subtle even in the standard case [44]. Additional complications are introduced by the slave bosons not being free to condense in arbitrary large number onto the lowest energy state, as well as by the fact that the binding of two bosons happens differently according to the number of dimensions. For lack of space we cannot go through the details, and we refer the reader to the literature $[39,45]$. Summarizing the features of the superconducting state from holon pairing as predicted by the RVB model, one has a gap in the single particle excitations, qualitatively like the BCS case, but possibly too small to be detected. The critical temperature is zero for the half-filled band case, then increases with the hole doping (contrary to the results of the fermionic version discussed in Sec. 2.4) and with the strength of the magnetic interaction.

The RVB model is far from being accepted by the scientific community, despite its ingenuity. The present autlıors are sceptical about its applicability in the case of IITCSC's. Indeed, to mention just one point of criticism, while the predicted increase in the perpendicular resistivity $\rho_{c}(T)$ as $1 / T$ on approaching $T_{\mathrm{c}}$ from above is crucial to the validity of the model, there is evidence that in good single crystals of YBCO [46] the presence or absence of a linear behaviour of $\rho_{\mathrm{c}}(T)$ down to $T_{\mathrm{c}}$ depends on the preparation condition. Thercfore the $1 / T$ trend seems to be an extrinsic effect, not connected to the intrinsic nature of the carriers. In single crystals of $\mathrm{La}_{x} \mathrm{Sr}_{x} \mathrm{CuO}_{4}$ the predicted rise of $\rho_{\mathrm{c}}\left(T \rightarrow T_{\mathrm{c}}^{+}\right)$is observed [47] for $x \leq 1.2$, but in those samples also the in-plane resistivity shows the same trend, contrary to RVB predictions. At, and above, the reported [47] optimal hole concentration for superconductivity both in-plane and out-of-plane resistivities decrease linearly as $T \rightarrow T_{\mathrm{c}}^{+}$. Also the predicted linearity of the normal state in-plane resistivity down to zero temperature has not been observed. Indeed, $\mathrm{EuBa}_{2} \mathrm{Cu}_{3} \mathrm{O}_{7}$ in the normal state induced by high magnetic field at few Kelvin reportedly shows [48] usual residual-resistivily behaviour. One can also mention that there is growing evidence from photoemission and other measurements that the ITCSC's present a well defined Fermi surface positioned in the reciprocal space as required by the Luttinger theorem. The superconducting state seems therefore to originate from a normal metallic phase whose basic features are those of a Fermi liquid.

\section{Acknowledgments}

This work was partly supported by C.N.R. through Progetlo Finalizzalo Tecnologie Superconduttive e Criogeniche. M.P. acknowledges a fellowship from Consorzio I.N.F.M., Progello Supercondultori ad Alla Temperalura. 


\section{References}

[1] Y.A. Izyumov, V.M. Laptev, Int. J. Mod. Phys. B 5, 563 (1991).

[2] J.R. Schrieffer, Theory of Superconductivity, W.A. Benjamin, New York 1964; P.G. de Gennes, Superconduclivily of Melals and Alloys, W.A. Benjamin, New York 1966; Superconductivity, Eds. M. Acquarone, S. Pace, World Scientific, Singapore 1991.

[3] S. Barisic, J. Labbé, J. Friedel, Phys. Rev. Lett. 25, 919 (1970).

[4] G.M. Eliashberg, Sov. Phys.-JETP 16, 780 (1963); D.J. Scalapino, in: Superconductivily, Vol. 1, Ed. R.D. Parks, Marcel Dekker, New York 1969, p. 449; P.B. Allen, B. Mitrovic, Theory of Superconducting $T_{c}$, Solid State Physics, Vol. 37, Eds. H. Ehrenreich, F. Seitz, D. Turnbull, Academic Press, New York 1982, p. 1.

[5] W.L. McMillan, Phys. Rev. 167, 331 (1968).

[6] M. Acquarone, in: Superconductivity, Eds. S. Pace, M. Acquarone, World Scientific, Singapore 1991.

[7] L. Pietronero, Europlys. Lett. 17, 365 (1992); L. Pietronero, S. Strassler, Phys. Rev. B, to appear.

[8] E. Marsch, Z. Phys. B 70, 279 (1988).

[9] E.W. Fenton, Solid State Commun. 65, 343 (1988); Phys. Rev. B 40, 10796 (1989).

[10] K. Mashida, M. Kato, Jpn. J. Appl. Phys. 26, 1660 (1987).

[11] L.L. Dacmen, A.W. Overhauser, Phys. Rev. B 39, 6431 (1989).

[12] D.J. Kï, Jpn. J. Appl. Phys. 26, L741 (1987).

[13] J.E. Hirsch, Phys. Rev. B 35, 8726 (1987).

[14] A.L. Kuzemsky, A. IIolas, N.M. Plakida, Plysica B 122, 168 (1983).

[15] F. Indurain, Solid Stale Commun. 81, 939 (1992); J. Bausells, F. Yndurain, J. Phys. C 17, 5539 (1984).

[16] A.N. Das, S. Sil, Physica C 161, 325 (1989); A.N. Das, J. Konior, D.K. Ray, Physica C 170, 213 (1990); A.N. Das, J. Konior, D.K. Ray, A.M. Oleś, Phys. Rev. B 44, 7680 (1991).

[17] J.R. Schriefter, X.G. Wen, S.C. Zhang, Phys. Rev. Lett. 60, 944 (1988) and Phys. Rev. Lett. 61, 2814 (1988); J.R. Schrieffer, X.G. Wen, S.C. Zhang, Phys. Rev. B 39, 11663 (1989); A. Kampf, J.R. Schrieffer, Phys. Rev. B 41, 6399 (1990); A. Kampf, J.R. Schrieffer, J. Phys. Chem. Solids 52, 1321 (1991).

[18] N.F. Mott, Philos. Mag. Lett. 64, 211, 217 (1991).

[19] For a review, see II. Kamimura, M. Eto, S. Matsuno, I. Ushio, Comments Condens. Maller Phys. 15, 303 (1992).

[20] M. Dayan, Solid State Commun. 67, 1073 (1988).

[21] R.J. Wijngaarden, R. Griessen, in: Sludics of High Temperature Superconductors, Vol. II, Ed. V. Narlikar, Nova Sci. Publ., New York 1990, p. 29; J.S. Schilling, S. Klotz, in: Physical Propertics of IIigh Temperature Superconductors, Vol. III, Ed. D.M. Ginsberg, World Scientific, Singapore 1992.

[22] E. Arrigoni, G. Strinati, Phys. Rev. B 44, 7455 (1991); and Phys. Rev. B, to appear.

[23] K.A. Chao, J. Spalek, A.M. Oleś, J. Phys. C 10, L27 (1977); J. Spatek, K.A. Chao, J. Phys. C 13, 5241 (1980).

[24] J.E. Hirsch, Phys. Rev. Letl. 54, 1317 (1985). 
[25] For a pedagogical presentation see e.g. M. Acquarone, in: The Physics of Melals, Ed. E.S. Giuliano, C. Rizzuto, World Scientific, Singapore 1988, p. 1.

[26] J. Spalek, A.M. Oleś, J.M. Honig, Phys. Rev. B 28, 6802 (1983); J. Spalek, A. Datta, J.M. Honig, Phys. Rev. B 33, 4891 (1986); J. Spalek, A. Datta, J.M. Honig, Phys. Rev. Lett. 59, 728 (1987).

[27] M. Gutzwiller, Phys. Rev. Lett. 10, 159 (1963). For a review see D. Vollhardt, Rev. Mod. Phys. 56, 99 (1984).

[28] J. Spałek, Phys. Rev. B 37, 533 (1988).

[29] M. Acquarone, Solid Slate Commun. 66, 937 (1988).

[30] R. Combescot, Phys. Rev. B 37, 2235 (1988).

[31] P.W. Anderson, Science 235, 196 (1987); G. Baskaran, Z. Zou, P.W. Anderson, Solid State Commun. 63, 973 (1987); P.W. Anderson, G. Baskaran, Z. Zou, T. Hsu, Phys. Rev. Lett. 58, 2790 (1987).

[32] P.W. Anderson, Mater. Res. Bull. 8, 153 (1973).

[33] F.D.M. Haldane, J. Phys. C 14, 2585 (1981); G. Baskaran, Inl. J. Mod. Phys. B 1, 3 (1988).

[34] A.E. Ruckenstein, P.J. Hirschfeld, J. Appel, Phys. Rev. B 36, 857 (1987); Y. Suzumura, Y. Hasegawa, H. Fukuyama, J. Phys. Soc. Jpn. 57, 2760 (1987); N. Nagaosa, P.A. Lee, Phys. Rev. B 54, 966 (1992).

[35] J.M. Luttinger, Phys. Rev. 119, 1153 (1960).

[36] S.A. Kivelson, J. Sethna, D. Roklisar, Phys. Rev. B 35, 8865 (1987).

[37] Z. Zou, P.W. Anderson, Phys. Rev. B 37, 627 (1988).

[38] P.W. Anderson, Z. Zou, Phys. Rev. Lett. 60, 132 (1988).

[39] J.M. Wheatley, T.C. Hsu, P.W. Anderson, Phys. Rev. B 37, 5897 (1988).

[40] J. Hubbard, Proc. R. Soc. Lond. A 285, 542 (1965).

[41] Y.A. Izyumov, B.M. Letfulov, J. Phys. Condens. Matler 2, 8905 (1990).

[42] G. Baskaran, Prog. Theor. Phys. Suppl. 107, 49 (1992).

[43] R. Frésard, P. Wolfle, Int. J. Mod. Pliys. B 6, 685 (1992).

[44] P. Nozières, D. Saint James, J. Phys. (Frince) 43, 1133 (1982).

[45] M.J. Rice, Y.R. Wang, Phys. Rev. B 37, 5893 (1988).

[46] G. Weingang, K. Winzer, Z. Phys. B 77, 11 (1989).

[47] T. Kimura, K. Kishio, T. Kobayashi, Y. Nakayama, N. Motohira, K. Kitazawa, K. Yamafuji, Physica C 192, 247 (1992).

[48] A. Yamagishi, H. Fuke, K. Sugiyama, M. Date, Y. Tajima, M. Hikita, T. Ishii, A. Katsui, Y. Hidaka, T. Iwata, S. Tsurumi, Physica C 153-155, 1459 (1988); Y. Tajima, M. Hikita, T. Ishii, II. Fuke, K. Sugiyama, M. Date, A. Yamagishi, A. Katsui, Y. Hidaka, T. Iwata, S. Tsurumi, Phys. Rev. B 37, 7956 (1988). 